\title{
Sharpness of the Phase Transition in Percolation Models *
}

\author{
Michael Aizenman ${ }^{\star \star}$ and David J. Barsky \\ Department of Mathematics, Rutgers University, New Brunswick, NJ 08903, USA
}

\begin{abstract}
The equality of two critical points - the percolation threshold $p_{H}$ and the point $p_{T}$ where the cluster size distribution ceases to decay exponentially is proven for all translation invariant independent percolation models on homogeneous $d$-dimensional lattices $(d \geqq 1)$. The analysis is based on a pair of new nonlinear partial differential inequalities for an order parameter $M(\beta, h)$, which for $h=0$ reduces to the percolation density $P_{\infty}$ - at the bond density $p=1-e^{-\beta}$ in the single parameter case. These are: (1) $M \leqq h \partial M / \partial h+M^{2}$ $+\beta M \partial M / \partial \beta$, and (2) $\partial M / \partial \beta \leqq|J| M \partial M / \partial h$. Inequality (1) is intriguing in that its derivation provides yet another hint of a " $\varphi^{3}$ structure" in percolation models. Moreover, through the elimination of one of its derivatives, (1) yields a pair of ordinary differential inequalities which provide information on the critical exponents $\hat{\beta}$ and $\delta$. One of these resembles an Ising model inequality of Fröhlich and Sokal and yields the mean field bound $\delta \geqq 2$, and the other implies the result of Chayes and Chayes that $\widehat{\beta} \leqq 1$. An inequality identical to (2) is known for Ising models, where it provides the basis for Newman's universal relation $\hat{\beta}(\delta-1) \geqq 1$ and for certain extrapolation principles, which are now made applicable also to independent percolation. These results apply to both finite and long range models, with or without orientation, and extend to periodic and weakly inhomogeneous systems.
\end{abstract}

\section{Introduction}

There have traditionally been two different notions of a critical point in percolation models, corresponding to the boundaries of the low density and the high density regimes. For the standard one parameter percolation models on the $d$ dimensional square lattice $\mathbb{Z}^{d}$ (i.e. site percolation or nearest neighboor bond

\footnotetext{
* Research supported in part by the NSF Grant PHY-8605164

$\star \star$ Also in the Physics Department
} 
percolation) the two critical densities are usually denoted by $p_{T}$ (or $\pi_{c}$ ) and, correspondingly, $p_{H}$ (or $p_{c}$ ). The main result reported here is the proof of the equality of these two critical points.

Expansion methods show that in the "high density" regime there is percolation (in two or more dimensional models, and in long range models in one dimension), while in the "low density" regime the cluster size distribution has exponential decay and the connectivity functions decay rapidly. Our main result is that these two phases extend up to a common critical point, without there being an intermediate phase in which with probability one there is no infinite cluster but the density of large cluster decays only by a power law.

The coincidence of the two critical points is proven here for general translation invariant independent models - which may include long range and/or oriented bonds - in any dimension.

Although such an assertion was expected by the physicists to be true - at least in the finite range case - previous mathematical treatments of the subject dealt successfully only with percolation models in dimensions $d=2$ and $d=1$. The former case included, of course, the celebrated proof of Kesten [1] for self similar models, and its extension by Russo [2] to other finite range two dimensional models. The reference to $d=1$ concerns, in addition to the trivial case of finite range models, also the rather special $1 /|x-y|^{2}$ models for which the coincidence of the critical points was derived in [3], by very different means. (Incidentally, the latter models exhibit also an intermediate phase, but within the high density regime [4].)

It may also be of interest to compare our results for percolation with the results of Aizenman [5] on the analogous problem for ferromagnetic Ising spin models. For the Ising (and related) spin systems, the equality of the high and low critical temperatures was proven only under a certain "regularity" hypothesis, whose verification required certain restrictions. The arguments presented here require no such hypothesis, and suggest that although the regularity is indispensable for some of the other results derived in [5], it is not so for the coincidence of the critical temperatures. ${ }^{1}$

Since translation invariance is the only assumption which is required here for independent systems, let us also remark that there are known examples of systems which are highly noninvariant for which $p_{T}$ does not equal $p_{H}$, Chayes and Chayes [6].

Before completing the summary of the results, and their relations with previous works, let us present the full definition of the two critical points $p_{T}$ and $p_{H}$, and introduce the notation we shall use for general independent percolation models. In the rest of the introduction we shall focus on (unoriented) bond percolation models, for which the length of the bonds need not be bounded. Oriented percolation, and partially oriented percolation (which we introduce in order to have a unified analysis), are discussed in Sect. 2.1. Site percolation and some other cases to which our analysis extends are discussed in Sects. 7 and 8.

${ }^{1}$ This result has now been derived and is presented in a companion paper, coauthored with Fernández [22] 


\subsection{The Setup}

The basic example of a percolation transition is observed in the nearest neighbor bond percolation model on the cubic lattice $\mathbb{L}=\mathbb{Z}^{d}$. For this model, bonds are pairs of neighboring lattice sites. Each bond $b=\{x, y\}$ has associated to it a random variable $n_{b}$ which can take one of the two values: 0 or 1 . If $n_{b}=1$, we refer to the bond $b$ as "occupied." The variables $\left\{n_{b}\right\}$ are jointly independent and hence their distribution is completely characterized by the density of the occupied bonds:

$$
p=\operatorname{Prob}\left(n_{b}=1\right) \text {. }
$$

For a given configuration of the bond variables we regard the occupied bonds as connecting, and decompose the set of lattice sites into the corresponding connected components. We denote by $C(x)$ the connected cluster of site $x \in \mathbb{L}$, and by $|C(x)|$ its size i.e., the number of lattice sites in it. Some key quantities of interest are

i) the expected size of the cluster containing a given site, say the origin 0 :

$$
\chi_{\text {total }}(p)=\langle|C(0)|\rangle \text {, }
$$

and ii) the probability that the origin belongs to an infinite cluster, i.e., the percolation density:

$$
M(p)=\operatorname{Prob}(|C(0)|=\infty) .
$$

The quantity $M(p)$ is more often denoted by $P_{\infty}$, which is consistent with the general notation for the cluster size distribution:

$$
P_{n}=\operatorname{Prob}(|C(0)|=n) \text {. }
$$

Well known arguments show that for small enough $p$ [in particular $\left.p<(2 d-1)^{-1}\right]$ the expected cluster size $\chi_{\text {total }}(p)$ is finite. Furthermore, the finiteness of $\chi_{\text {total }}(p)$ is a good criterion for the low density phase. For example, it is known to imply exponential decay of the cluster size distribution $[7,8]$.

At the other extreme, for $p$ close enough to 1 , not only does $\chi_{\text {total }}(p)$ diverge, but also there is a positive probability that the origin belongs to an infinite cluster $(M(p)>0)$. For such values of $p$ the probability of generating an infinite cluster somewhere on the lattice $\mathbb{L}-$ is one.

The two critical densities to which we referred above, are the boundaries of these high and low density regimes. Their natural definitions are

$$
p_{T} \equiv \pi_{c}=\sup \left\{p \mid \chi_{\text {total }}(p)<\infty\right\}
$$

and

$$
p_{H} \equiv p_{c}=\inf \{p \mid M(p)>0\}
$$

Obviously, $p_{T} \leqq p_{H}$.

In the more general class of independent bound percolation models, any two sites may be directly connected by an occupied bond; however, the probability of bonds to be occupied decreases for long bonds. Thus the set of bonds consists of all pairs of lattice sites, and with each such pair $b=\{x, y\}$ we have associated an occupation random variable $n_{b}$. The bonds are occupied independently with the 
translation invariant probabilities

$$
\operatorname{Prob}\left(n_{b}=1\right)=K_{b}=1-e^{-\beta J_{x, y}},
$$

for $b=\{x, y\}$.

The parameter $\beta(\geqq 0)$, which is in a way redundant, is introduced both for mathematical convenience and because it allows the analogies with ferromagnetic spin models to be seen more clearly. It is most convenient to study the percolation transition by varying $\beta$ at fixed values of the parameters $J_{x, y}(\geqq 0)$. The critical values $\beta_{T}$ and $\beta_{H}$ are defined by the same criteria as $p_{T}$ and $p_{H}$ in (1.5).

It should be noted that the class of percolation models described by (1.6) includes long range models, for which $J_{x, y}$ decays slowly, e.g. $J_{x, y} \cong 1 /|x-y|$. While finite range models exhibit percolation only in dimensions $d \geqq 2$, such long range systems may percolate even in one dimension (iff $s \leqq 2$ [9]). To avoid models with $\beta_{H}=0$, we assume that

$$
|J| \equiv \sum_{x} J_{0, x}<\infty
$$

The results in this paper apply to all such systems.

Other independent percolation models covered by our analysis include oriented percolation - for which one has only to reinterpret the notions used in our discussion of ordinary bond percolation (see Sect. 2.1) - and site percolation for which the adjustments are found in Sect. 7. Furthermore, one may replace the translation invariance requirement with periodicity or even weaker conditions (see Sect. 8.1).

\subsection{The Main Results}

Our main results are the following two propositions. They apply to the percolation transition which is observed by varying one of the parameters of the model. The parameter is either $p$, as in (1.1), or $\beta$ for the more general case derived by (1.6). For the former case no other parameter was introduced; however, in the more general case we also could have chosen to look at the transition produced by varying the bond densities of bonds $\{x, y\}$ with given values of $y-x$. The results would be the same.

Theorem 1.1. For any independent translation invariant bond, or site, percolation model on $\mathbb{Z}^{d}$ (like the bond models introduced above),

$$
\beta_{T}=\beta_{H} \quad \text { or, when applicable, } p_{T}=p_{H} .
$$

We shall denote the common value in (1.8) by $\beta_{c}$ (or $p_{c}$ ). To state the second result, which is in fact used in the proof of Theorem 1.1, let us define [extending (1.3)], for $h \geqq 0$,

$$
M(\beta, h)=1-\sum_{1 \leqq n<\infty} P_{n}(\beta) e^{-n h} .
$$

The quantity $M$ is of interest because it contains the Laplace transform of the cluster size distribution, because of its analogy with the spontaneous magnetization in ferromagnetic models, and because of its utility in the derivation of the above result. 
Theorem 1.2. Under the same assumptions as in Theorem 1.1 (independence and translation invariance),

$$
M\left(\beta_{T}, h\right) \geqq \text { const } h^{1 / 2} .
$$

Let us remark here that, making sufficiently strong assumptions on the existence of power laws, it is customary to define the critical exponent $\delta$ by the relations

$$
M\left(\beta_{T}, h\right) \cong h^{1 / \delta}, \quad \text { or } \quad P_{\geqq n}\left(\beta_{T}\right) \equiv \sum_{m \geqq n} P_{m} \cong n^{-1 / \delta} .
$$

For the sake of concreteness, we shall define $\delta$ by:

$$
\delta=\liminf _{h \succ 0} \frac{\ln h}{\ln M\left(\beta_{T}, h\right)} .
$$

The result (1.10) shows that quite generally this exponent obeys the mean field bound:

$$
\delta \geqq 2,
$$

which is in fact saturated $(\delta=2)$ in the case of percolation models on a Bethe lattice.

The proofs of these two theorems are based on a number of differential inequalities which are also of independent interest. In particular, we prove that for bond percolation models, in the generality described above, the quantity $M$ satisfies:

$$
\frac{\partial M}{\partial \beta} \leqq|J| M \frac{\partial M}{\partial h},
$$

and

$$
M \leqq h \frac{\partial M}{\partial h}+M^{2}+\beta M \frac{\partial M}{\partial \beta}
$$

(in the weak sense, as explained in Sect. 3). These inequalities are closely related to previous results of other works on percolation and ferromagnetic systems. We shall mention some of those below. Let us first however make some additional remarks on the nature of these inequalities.

The differential inequality (1.14) is identical to one obeyed by the magnetization in ferromagnetic Ising spin systems, for which it follows from the GriffithsHurst-Sherman inequality [10]. Newman has pointed out that (1.14) bears an amusing relation to the Burgers equation, and furthermore, that it implies the critical exponent inequality [11]:

$$
\widehat{\beta}(\delta-1) \geqq 1
$$

for models with $\beta_{T}=\beta_{H}$. Here $\hat{\beta}$ is the critical exponent which enters in the expected power law:

$$
M\left(\beta_{H}+t, 0\right) \equiv P_{\infty}\left(\beta_{H}+t\right) \cong t_{+}^{\hat{\beta}} .
$$

Newman's observation was extended in [12] to a number of useful extrapolation principles, which are now made applicable also to percolation (see Sect. 6). It is 
interesting to note that the inequality (1.16) is saturated in both percolation and ferromagnetic systems by the mean field (or Bethe lattice) values of the two critical exponents - despite the fact that individually these exponents take on different values in the two systems.

The relation (1.15) is truly remarkable. A coarse glance at its derivation shows that hidden in it are some key features of percolation models which (like the previous work of [13] and the rigorous results of [8]) indicate a relation with a " $\varphi$ " " field theory. Furthermore, combined with more general properties of the "order parameter" $M$ [like (1.14)], the inequality (1.15) unifies previous results which dealt separately with the regimes: $R_{1}=\left\{\beta=\beta_{T}, h>0\right\}$ and $R_{2}=\left\{\beta>\beta_{H}, h=0\right\}$, and leads also to a proof of the equality $\beta_{T}=\beta_{H}$.

Our derivation of the inequality (1.15) grew out of an attempt to understand the relation between the recent result of Chayes and Chayes [14], who proved (under a harmless assumption)

$$
P_{\infty}(p) \leqq P_{\infty}(p)^{2}+P_{\infty}(p) \frac{\partial P_{\infty}(p)}{\partial p},
$$

and the inequality

$$
\left|\frac{\partial}{\partial h}\left(\frac{M}{h}\right)\right| \leqq|K|\left(\frac{M}{h}\right)^{3}+\frac{M}{h},
$$

which we had previously derived by means which are very different from the proof given below. Here inequality (1.18) is presented in its site percolation version and $|K|=\sum_{x} K_{\{0, x\}}$ [see (1.6)]. In the original derivation of (1.19), which may equivalently be stated as

$$
M \leqq h \frac{\partial M}{\partial h}+\frac{M}{h}\left(h^{2}+|K| M^{2}\right),
$$

we were guided by the analysis of [8] and by the recent work of Fröhlich and Sokal $[15,16]$ on ferromagnetic spin models, for which they proved

$$
M \leqq h \frac{\partial M}{\partial h}+\frac{M^{2}}{h}(h+|J| M)^{2} .
$$

The differential inequality (1.19) is readily integrated and leads to the proof of Theorem 1.2, whereas (1.18) was used by Chayes and Chayes [14] for the derivation of the critical exponent mean field bound:

$$
\hat{\beta} \leqq 1 \text {. }
$$

It may be noted that (1.16) and (1.22) imply (1.13) - but only when it is known that $\beta_{T}=\beta_{H}$. The very versatile differential inequality (1.15) implies inequalities having all the essential characteristics of (1.18) and (1.20), and moreover, it leads to the proof of the equality of the two critical points.

The outline of the paper is as follows. In Sect. 2 we generalize the setup to include oriented percolation, and introduce a useful ghost field representation. The main inequalities are derived in Sect. 3, and their applications are found in Sects. 4-6. Site percolation and other extensions of the results are found in Sects. 7 and 8. 


\section{Extension of the Setup}

Our purpose is to introduce here three different notions. The first is a generalization of bond percolation models which allows us to treat simultaneously unoriented and oriented percolation. Readers interested solely in the former may skip this discussion (Sect. 2.1). Next is the "ghost field" representation which is used to give a convenient probabilistic interpretation of the order parameter $M(\beta, h)$. Finally, we describe the natural finite volume approximations.

\subsection{Partially Oriented Percolation}

This paper is written in such a way that it is possible to read it having either regular (unoriented) or oriented percolation in mind. To facilitate this, we shall generalize the setup introduced in Sect. 1.2, extending it to partially oriented models - which include both oriented and unoriented bonds. Such systems were first considered by Hammersley [7].

In some oriented bond percolation models, bonds are regarded as ordered rather than unordered pairs of sites. When the bond $b=(x, y)$ is occupied $\left(n_{b}=1\right)$, there is a connection from $x$ to $y(x \rightarrow y)$ without there being any implication for the connection from $y$ to $x$. As before, the bonds are independently occupied with the translation invariant probabilities,

$$
\operatorname{Prob}\left(n_{b}=1\right)=1-e^{-\beta J_{x \rightarrow y}},
$$

for $b=(x, y)$. A given pair of sites $\{x, y\}$ may participate in two bonds, $(x, y)$ and $(y, x)$, which are occupied independently and for which the couplings $J_{x \rightarrow y}$ and $J_{y \rightarrow x}$ need not be equal. The cluster of $x, C(x)$, is now the set of all sites to which $x$ is connected: the sites which can be reached from $x$ by following a path of occupied bonds in the direction allowing a connection.

An example of such a system is the oriented nearest neighbor bond percolation model on $\mathbb{Z}^{d}$ for which the projection of $x \in \mathbb{Z}^{d}$ on the vector $(1,1, \ldots, 1) / \sqrt{d}$ is regarded as the time coordinate. For this model

$$
J_{x \rightarrow y}=\left\{\begin{array}{ll}
1 & \text { if } \quad|x-y|=1 \\
0 & \text { otherwise. }
\end{array} \text { and } \sum_{i=1}^{d}\left(y_{i}-x_{i}\right)>0,\right.
$$

Let us now describe what we shall refer to as partially oriented percolation models. These are obtained by associating with each pair of sites three bonds: one of which is labelled by the unoriented pair $\{x, y\}$, and the other two by the two oriented pairs, $(x, y)$ and $(y, x)$. The first, when occupied, connects both $x$ to $y$ and $y$ to $x$, while the bond $(x, y)$ is regarded as oriented - connecting only $x$ to $y$. The bonds are occupied independently, with

$$
\operatorname{Prob}\left(n_{\{x, y\}}=1\right)=\operatorname{Prob}(\{x, y\} \text { is occupied })=1-e^{-\beta J_{x, y}},
$$

and

$$
\operatorname{Prob}\left(n_{(x, y)}=1\right)=\operatorname{Prob}((x, y) \text { is occupied })=1-e^{-\beta J_{x \rightarrow y}},
$$


where $J_{x, y}, J_{x \rightarrow y}$, and $J_{y \rightarrow x}$ are regarded as separate parameters (and in general are not equal). However, we will assume that the couplings $J$ are translation invariant, meaning that for all $x, y$, and $z$ :

$$
J_{x+z, y+z}=J_{x, y} \text {, and } J_{x+z \rightarrow y+z}=J_{x \rightarrow y} .
$$

A path from the site $x$ to the site $y$ is a sequence of bonds whose arrangement satisfies the obvious incidence relations. That is, the bond $(u, v)$ may appear only in the step which proceeds from $u$ to $v$ and not vice versa, whereas $\{u, v\}$ may be used in both directions.

The cluster of $x, C(x)$, is now defined (for a given bond configuration) to be the set of all sites $y$ for which there exists a path of occupied bonds from $x$ to $y$. (Note that the set of clusters of the sites of $\mathbb{Z}^{d}$ no longer offers a disjoint decomposition of the lattice.)

With this definition of the cluster we continue to define the quantities $P_{n}$ by (1.4). The expected cluster size $\chi_{\text {total }}$ and the order parameter $M$ are still given by (1.2) and (1.9). Finally, we modify the symbol $|J|$ to mean:

$$
|J|=\sum_{x}\left(J_{0, x}+J_{0 \rightarrow x}\right) .
$$

Obviously, the unoriented models described in the introduction may be regarded as special partially oriented models, with $J_{x \rightarrow y} \equiv 0$. However, it is also the case that a reader interested in only the more standard (unoriented) models may read the rest of the paper ignoring the fact that our notions were generalized in this subsection.

\subsection{The Ghost Field Representation for $M(\beta, h)$}

We shall now introduce a construction which provides a useful probabilistic interpretation of the order parameter $M(\beta, h)$ - extending the intuition one has for $P_{\infty}$.

For that purpose, we augment the model by adding to it random site variables $\left\{m_{x}\right\}_{x \in \mathbb{Z}^{d}}\left(m_{x}=0\right.$, or 1$)$. Probabilities are assigned so that $\left\{n_{b}, m_{x}\right\}$ forms a jointly independent set of random variables, with

$$
\operatorname{Prob}\left(m_{x}=1\right)=1-e^{h} \quad(\cong h, \text { for } h \text { small })
$$

for every $x \in \mathbb{Z}^{d}$, and with $\operatorname{Prob}\left(n_{b}=1\right)$ still given by (1.6) or (2.1).

For a given configuration $\left\{n_{b}, m_{x}\right\}$ of bond and site variables, we denote by $G$ the set of sites $x$ with $m_{x}=1$ and call these sites "green." (These are sometimes referred to as "ghost" sites, as in $[17,12]$.) We continue to define the connected cluster of $x$ as above and we say that $x$ is connected to the green set if at least one site in $C(x)$ is green.

Proposition 2.1. For the augmented system defined above, with $h>0$,

$$
\operatorname{Prob}(C(0) \cap G \neq \emptyset) \equiv \operatorname{Prob}(0 \text { is connected to } G)=M(\beta, h),
$$

where $M$ is the order parameter defined in (1.9). 
Proof. First observe that for any nonrandom set $A \subset \mathbb{Z}^{d}$,

$$
\operatorname{Prob}(A \cap G \neq \emptyset)=1-\prod_{x \in A} e^{-h}=1-e^{-h|A|},
$$

where $|A|=\operatorname{card}(A)$.

On the left-hand side of (2.5), condition on the cluster of the origin:

$$
\operatorname{Prob}(C(0) \cap G \neq \emptyset)=\sum_{A \subset \mathbb{Z}^{d}} \operatorname{Prob}(A \cap G \neq \emptyset \mid A=C(0)) \operatorname{Prob}(A=C(0)) \text {. }
$$

Note that the events " $A \cap G \neq \emptyset$ " and " $A=C(0)$ " are independent, as the first depends only on site variables and the second depends only on bond variables. With (2.6), we obtain

$$
\operatorname{Prob}(C(0) \cap G \neq \emptyset)=\sum_{A \subset \mathbb{Z}^{d}} \operatorname{Prob}(A=C(0))\left(1-e^{-h|A|}\right) .
$$

Combining this relation with the fact [see (1.4)] that

$$
\sum_{A:|A|=n} \operatorname{Prob}(A=C(0))=P_{n}
$$

yields

$$
\operatorname{Prob}(C(0) \cap G \neq \emptyset)=\sum_{1 \leqq n \leqq \infty} P_{n}\left(1-e^{-n h}\right)=1-\sum_{n=1}^{\infty} P_{n} e^{-n h} .
$$

The $n=\infty$ term would make no contribution to the sum on the right-hand side (since $h>0$ ) and for this reason is not included.

Although (2.5) is valid only for positive values of $h, M(\beta, h)$ is defined by (1.9) also for $h=0$ and, in fact, $M(\beta, 0)=P_{\infty}(\beta)$. Since $M$ is clearly continuous from the right at $h=0$, Proposition 2.1 shows also that

$$
\lim _{h \backslash 0} \operatorname{Prob}(C(0) \cap G \neq \emptyset)=P_{\infty} .
$$

This limit suggests that the green sites may be regarded as surrogates for the point at infinity. The green sites evacuate every finite region in $\mathbb{Z}^{d}$ as $h$ vanishes. In this sense, the event "the origin is connected to the green set" is a small $h$ approximation to the event "the cluster of the origin is infinite."

Because of certain analogies with Ising spin systems, we denote the order parameter by $M$, the symbol used for the magnetization in those models. The independent variable $h$ plays the role of an external magnetic field. As in the case of Ising models, we define the susceptibility to be the derivative of $M$ with respect to $h$ :

$$
\chi(\beta, h)=\frac{\partial M(\beta, h)}{\partial h}=\sum_{n=1}^{\infty} n P_{n} e^{-n h}, \quad h>0 .
$$

The definition of $\chi$ is extended to $h=0$ by continuity:

$$
\chi(\beta, 0)=\lim _{h \downarrow 0} \chi(\beta, h)=\sum_{n=1}^{\infty} n P_{n}=\langle|C(0)| \cdot I[|C(0)|<\infty]\rangle,
$$

where $I[-]$ is the indicator function which vanishes when the cluster is infinite. 
For $\beta<\beta_{H}$, infinite clusters have zero probability of existing. Hence the expected sizes of clusters and finite clusters coincide:

$$
\chi(\beta, 0)=\chi_{\text {total }}(\beta) \equiv\langle|C(0)|\rangle .
$$

It is desirable to develop a geometric interpretation for the susceptibility as well as the order parameter. This is accomplished in the next statement.

Proposition 2.2. For any $h>0$,

$$
\chi=\langle|C(0)| \cdot I[C(0) \cap G=\emptyset]\rangle .
$$

Proof. Since the distribution of the green sites is independent of the bond variables, it is convenient to evaluate the right-hand side of (2.9) by first conditioning it on the bond configuration. The conditional expectation of the indicator function is exactly $e^{-h|C(0)|}$, and hence

$$
\langle|C(0)| \cdot I[C(0) \cap G=\emptyset]\rangle=\left\langle|C(0)| e^{-h|C(0)|}\right\rangle=\sum_{n} n P_{n} e^{-n h}=\chi .
$$

Remarks. 1) As the above argument shows, for positive $h$ there is no contribution in (2.9) from the event that the cluster of the origin is infinite. For an expression which is valid also at $h=0$, one should modify the right-hand side of (2.9) by explicitly adding the restriction that $|C(0)|<\infty$.

2) The following is a simple but convenient trick which is frequently of use. Since

$$
|C(0)|=\sum_{x} I[x \in C(0)],
$$

we have by Proposition 2.2 that for $h>0$

$$
\chi=\sum_{x}\langle I[x \in C(0)] I[C(0) \cap G=\emptyset]\rangle .
$$

Observe that the summand in (2.10) converges in the $h \searrow 0$ limit to the probability that the origin is connected to $x$ and the cluster of the origin is finite. This is one of the natural notions of the truncated two point connectivity function.

\subsection{The Finite Volume Approximation}

Here we introduce the finite volume quantities and state their basic relation with the corresponding functions for the infinite lattice. The reasons for considering finite systems are:

(i) all of the quantities of interest (in particular the order parameter) are trivially analytic in $\beta$,

(ii) the derivations of the main inequalities (1.14) and (1.15) rely on Russo's formula, the application of which is immediately valid only on a finite lattice, and

(iii) the proof of (1.15) additionally uses the van den Berg-Kesten inequality which also has an initial finite lattice requirement.

In order to maintain translation invariance at finite volume, we shall study percolation on the cubes $\Lambda_{L}=(-L, L]^{d} \cap \mathbb{Z}^{d}$ with periodic boundary conditions. The periodic models are defined by setting the bond occupation probabilities, for $x, y \in \Lambda_{L}$, to be

$$
\operatorname{Prob}_{L}\left(n_{\{x, y\}}=1\right)=1-e^{-\beta J_{x, y}^{(L)}}
$$


with

$$
J_{x, y}^{(L)}=\sum_{z \in \mathbb{Z}^{d}} J_{x, y+2 L z}
$$

and similarly for the oriented bonds with the finite volume oriented couplings $J_{x \rightarrow y}^{(L)}$.

Remark. There are also other natural boundary conditions which may be imposed on $\Lambda_{L}$ (e.g., the wired and free boundary conditions discussed in [18]) but they have the disadvantage of lacking homogeneity.

We let

$$
P_{n}^{(L)}=P_{n}^{(L)}(\beta)=\operatorname{Prob}_{L}(|C(0)|=n),
$$

and define the finite volume order parameter and susceptibility by

$$
M_{L}(\beta, h) \equiv 1-\sum_{n=1}^{\infty} P_{n}^{(L)}(\beta) e^{-n h}
$$

and

$$
\chi_{L}(\beta, h) \equiv \sum_{n=1}^{\infty} n P_{n}^{(L)}(\beta) e^{-n h} \quad\left(=\frac{\partial M_{L}}{\partial h}\right) .
$$

The finite volume order parameter and susceptibility have probabilistic interpretations similar to those of their full volume analogues. As in the preceding section, it is easily verified that

$$
M_{L}=\operatorname{Prob}_{L}(C(0) \cap G \neq \emptyset) .
$$

That is, Proposition 2.1 holds at finite volume. Likewise, a finite volume positive $h$ version of Proposition 2.2 can be proved by repeating the arguments of Sect. 2.1. In particular, we obtain the identity

$$
\chi_{L}=\sum_{x \in \Lambda_{L}} \operatorname{Prob}_{L}(x \in C(0), C(0) \cap G=\emptyset) .
$$

Information about finite volume quantities is translated into information about full volume quantities by taking the "infinite volume limit."

Proposition 2.3. For any $\beta \geqq 0$ and $h>0$,

$$
M_{L}(\beta, h) \underset{L \rightarrow \infty}{\longrightarrow} M(\beta, h),
$$

and

$$
\chi_{L}(\beta, h) \underset{L \rightarrow \infty}{\longrightarrow} \chi(\beta, h)
$$

Remark. For $h=0,(2.18)$ certainly fails if $\beta>\beta_{H}$. If in addition $\chi(\beta, 0)<\infty,(2.19)$ also fails to hold.

These claims may be regarded as well known. Still, for the sake of completeness, and because of the fact that we refer now to more general models, we shall give a proof of these assertions in the Appendix. However, let us comment here that some of the more natural arguments which can involve a "free energy" 
and convexity considerations for the proof of Lemma 2.3 in the unoriented case, are not applicable to the more general partially oriented percolation models.

We now list a number of useful properties of the order parameter. The first of these follows directly from the definitions of $M(1.9)$ and $M_{L}(2.14)$.

Lemma 2.4. Let $\beta>0$ be fixed. Then $M$ and $M_{L}$ are increasing, concave and analytic functions of $h$, for $h>0$.

We next consider the $\beta$-dependence of $M(\beta, h)$. When $h=0$, we have $M(\beta, 0)$ $=P_{\infty}(\beta)$, and thus $M(\beta, 0)$ is clearly nondecreasing in $\beta$. In fact a stronger statement, which is a consequence of Proposition 2.1 and its finite volume version (2.16) is true.

Lemma 2.5. At any fixed value of $h(\geqq 0), M$ and $M_{L}$ are nondecreasing functions of $\beta$.

The question of the analyticity of the order parameter is more difficult to resolve at the level of generality considered here. The following lemma summarizes some of what is known in this direction. For proofs, see the Appendix. (These results will not be used in this paper.)

Lemma 2.6. (i) $M$ is a continuous function of $\beta$ for $h>0$ and all $\beta \geqq 0$ and - in the unoriented case - also for $h=0$ and $\beta \neq \beta_{H}$.

(ii) In finite range percolation models, $M$ is an analytic function of $\beta$ for $h>0$ and $\beta>0$.

\section{Derivation of the Main Differential Inequalities}

In this section we consider the finite volume periodic models introduced in the preceding section and prove the differential inequalities upon which Theorems 1.1 and 1.2 rest. In subsequent sections we will integrate these inequalities at finite volume and then take the infinite volume limit. The two inequalities obtained and employed in this manner are found in the following propositions.

Proposition 3.1. In a finite volume periodic model with $M_{L}$ and $\chi_{L}$ defined by (2.14) and (2.15),

$$
\frac{\partial M_{L}}{\partial \beta} \leqq|J| M_{L} \chi_{L}
$$

Proposition 3.2. With the same hypotheses as above,

$$
M_{L} \leqq h \chi_{L}+M_{L}^{2}+\beta M_{L} \frac{\partial M_{L}}{\partial \beta} .
$$

Remarks. i) The quantity $|J|$ enters (3.1) as $\sum_{x \in \Lambda_{L}}\left(J_{0, x}^{(L)}+J_{0 \rightarrow x}^{(L)}\right)$, which by our choice of finite volume couplings in (2.12) equals $\sum_{x \in \mathbb{Z}^{d}}\left(J_{0, x}+J_{0 \rightarrow x}\right) \equiv|J|$ [see (2.3)].

ii) The above inequalities are finite volume versions of (1.14) and (1.15). In view of Proposition 2.3 the inequalities extend trivially to distributional inequalities for 
the infinite volume order parameter $M(\beta, h)$, for $h>0$. (Note that for this purpose, in (3.2) one should rewrite $M_{L} \frac{\partial M_{L}}{\partial \beta}$ as $\frac{1}{2} \frac{\partial M_{L}^{2}}{\partial \beta}$.) When $M$ is differentiable (see Lemmas 2.4 and 2.6), those inequalities are valid in the classical sense. The above observation could be used for an alternative direct derivation of the infinite volume results reported here. We shall however use (3.1) and (3.2) in the more explicit way outlined at the beginning of this section.

\subsection{Proof of Proposition 3.1}

Before stating the differentiation formula which is used, let us introduce some technical terms.

Definition. The event $E$ is increasing with respect to the bond variables $\left\{n_{b}\right\}$ (or the site variables $\left.\left\{m_{x}\right\}\right)$ on $\Lambda_{L}$ if the indicator function $I[E]$ is nondecreasing in $n_{b}$ (respectively, $m_{x}$ ) for every bond $b$ (respectively, site $x$ ) in $\Lambda_{L}$.

An important example of an event which is increasing in both the bond and site variables is the event that the origin is connected to the green set: $C(0) \cap G \neq \emptyset$.

Definition. For a given configuration $\left\{n_{b}, m_{x}\right\}$, the bond $b_{0}$ is called pivotal for the event $E$ if $E$ occurs for $\left\{n_{b}^{(1)}, m_{x}\right\}$ but not for $\left\{n_{b}^{(0)}, m_{x}\right\}$. Here $\left\{n_{b}^{(i)}\right\}(i=1,0)$ are the bond configurations obtained from $\left\{n_{b}\right\}$ by requiring the bond $b_{0}$ to be occupied $(i=1)$ or unoccupied $(i=0)$. Note that the event that $b_{0}$ is a pivotal bond is independent of the status of its occupation.

With these definitions, we can now state a useful differentiation formula.

Proposition 3.3 (Russo's formula [2]). If the event $E$ is increasing with respect to the bond variables on $\Lambda_{L}$, then for each bond $b_{0}$,

$$
\begin{aligned}
\frac{\partial \operatorname{Prob}_{L}(E)}{\partial p_{b_{0}}} & =\operatorname{Prob}_{L}\left(b_{0} \text { is pivotal for } E\right) \\
& =\frac{1}{1-p_{b_{0}}} \operatorname{Prob}_{L}\left(n_{b_{0}}=0, b_{0} \text { is pivotal for } E\right),
\end{aligned}
$$

where

$$
p_{b_{0}}=\operatorname{Prob}_{L}\left(n_{b_{0}}=1\right) .
$$

Proof of Proposition 3.1. Recalling (2.16) and applying the Chain Rule followed by Russo's formula yields

$$
\frac{\partial M_{L}}{\partial \beta}=\sum_{b} J_{b}^{(L)} \operatorname{Prob}_{L}\left(n_{b}=0, \text { the bond } b \text { is pivotal for " } C(0) \cap G \neq \emptyset\right. \text { "), }
$$

where the summation is over all the oriented and unoriented bonds, which we uniformly denote by $b$. The couplings are $J_{b}^{(L)}=J_{x, y}^{(L)}$ for the unoriented bonds, 


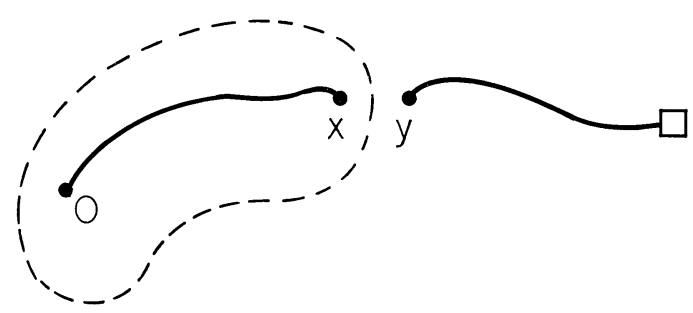

Fig. 1. The event that $n_{\{x, y\}}=0$ and $\{x, y\}$ is pivotal for “ $C(0) \cap G \neq \emptyset$ " up to an $x \leftrightarrow y$ permutation. The solid lines show connections made by paths of occupied bonds, the square symbolizes the green set, and the dashed line represents the hypersurface which demarcates the cluster of the origin. Every bond which could connect sites on the inside of the surface to sites on the outside is unoccupied

$b=\{x, y\}$, and $J_{b}^{(L)}=J_{x \rightarrow y}^{(L)}$ for the oriented bonds, $b=(x, y)$. (In unoriented models, the latter couplings are identically zero.)

Suppose that the unoriented bond $b_{0}$ is unoccupied and pivotal for the origin being connected to the green set. Then the origin is connected to precisely one of the two sites of $b_{0}$, the other site is connected to the green set and the cluster of the origin is free of green sites (see Fig. 1).

If the bond $b_{0}=(x, y)$ is oriented but otherwise as above, then we see that the same line of reasoning applies. Moreover, in this situation we know that $x$ is the site to which the origin is connected.

In light of these considerations, (3.3) can be rewritten as

$$
\frac{\partial M_{L}}{\partial \beta}=\sum_{x, y \in \Lambda_{L}} \operatorname{Prob}_{L}(x \in C(0), C(0) \cap G=\emptyset, C(y) \cap G \neq \emptyset)\left[J_{x, y}^{(L)}+J_{x \rightarrow y}^{(L)}\right] .
$$

Partitioning the events on the right-hand side of (3.4) according to the cluster of the origin, we obtain

$$
\begin{aligned}
\frac{\partial M_{L}}{\partial \beta} & =\sum_{x, y \in \Lambda_{L}} \sum_{A \subset \Lambda_{L}} \operatorname{Prob}_{L}(A=C(0), x \in C(0), A \cap G=\emptyset, C(y) \cap G \neq \emptyset)\left[J_{x, y}^{(L)}+J_{x \rightarrow y}^{(L)}\right] \\
& =\sum_{x, y \in A_{L}} \sum_{A: x \in A} \operatorname{Prob}_{L}\left(A=C(0), A \cap G=\emptyset, C_{A_{L} \backslash A}(y) \cap G \neq \emptyset\right)\left[J_{x, y}^{(L)}+J_{x \rightarrow y}^{(L)}\right] .
\end{aligned}
$$

If $y$ is in $\Lambda_{L} \backslash A$, then $C_{\Lambda_{L} \backslash A}(y)$ is the cluster of sites to which $y$ is connected by a path of occupied bonds none of whose sites lies in $A$. For $y$ not in $\Lambda_{L} \backslash A, C_{A_{L} \backslash A}(y)$ is defined to be the empty set. The second equality in (3.5) is obtained by noting that a consequence of $y$ lying outside of $C(0)$ [since $y$ is connected to $G$ and $C(0)$ contains no green sites] is that the path connecting $y$ to $G$ cannot make use of any bond having a site in $C(0)$.

In the terminology of [8], $C(0)$ is a self-determined random subset of $\Lambda_{L}$. Selfdetermined sets are often employed in the analysis of percolation models because they possess a locality property reminiscent of the nonanticipatory feature of stopping times in Markov processes and martingales. We make use of this property in the following way. Since all of the bonds which permit connections 
from sites in $C(0)$ to sites out of $C(0)$ are unoccupied, the cluster does not connect to the remainder of $\Lambda_{L}$. Thus the distribution of bonds having no sites in $A$ is unchanged when conditioned on the event " $A=C(0)$." For this reason, (3.5) becomes

$$
\begin{aligned}
\frac{\partial M_{L}}{\partial \beta}= & \sum_{x, y \in A_{L}} \sum_{A: x \in A} \operatorname{Prob}_{L}(A=C(0), A \cap G=\emptyset) \\
& \times \operatorname{Prob}_{L}\left(C_{A_{L} \backslash A}(y) \cap G \neq \emptyset\right)\left[J_{x, y}^{(L)}+J_{x \rightarrow y}^{(L)}\right] .
\end{aligned}
$$

Clearly, the probability that $y$ is connected to the green set in $\Lambda_{L} \backslash A$ is bounded above by the probability that $y$ is connected to the green set in all of $A_{L}$, i.e., by the finite volume order parameter. Thus, performing the summation over the sets $A$ in the above expression, we have

$$
\frac{\partial M_{L}}{\partial \beta} \leqq M_{L} \sum_{x \in \Lambda_{L}} \operatorname{Prob}_{L}(x \in C(0), C(0) \cap G=\emptyset) \sum_{y \in \Lambda_{L}}\left[J_{x, y}^{(L)}+J_{x \rightarrow y}^{(L)}\right] .
$$

The proof is completed by using (2.3), (2.12), and (2.17) to re-express the righthand side of this bound.

\subsection{Proof of Proposition 3.2}

Let us now introduce the inequality of van den Berg and Kesten [19] which we will use in the proof of Proposition 3.2.

Definition. Let $E_{1}$ and $E_{2}$ be a given pair of events in a random bond/site model. We say that the events $E_{1}$ and $E_{2}$ occur disjointly, for a particular configuration $k=\left\{n_{b}, m_{x}\right\}$, if there exist two disjoint bond/site collections $A_{1}$ and $A_{2}$ such that $E_{i}$ occurs for any configuration which agrees with $k$ on $A_{i}(i=1,2)$. The event which is represented by the set of all such configurations is denoted by $E_{1} \circ E_{2}$.

Proposition 3.4 (van den Berg-Kesten inequality [19]). For a bond/site model with independent random variables, if $E_{1}$ and $E_{2}$ are equally monotone in the occupation variables, then

$$
\operatorname{Prob}_{L}\left(E_{1} \circ E_{2}\right) \leqq \operatorname{Prob}_{L}\left(E_{1}\right) \operatorname{Prob}_{L}\left(E_{2}\right) .
$$

In the particular case that $E$ is the event that a given site $x$ is connected to the green set, $E \circ E$ is the event that $x$ is doubly connected to the green set. Since this notion will play a key role in this section, let us define it explicitly.

Definition. The site $x$ is doubly connected to the green set if there exist two distinct green sites $g$ and $g^{\prime}$ such that $x$ is connected to those sites by a pair of paths of occupied bonds which can be chosen so as to not share a common bond. (If $x$ is green, one of these paths may be the empty path.)

In the proof of Proposition 3.2 we shall use (following [14]) a particular decomposition of the event " $C(0) \cap G \neq \emptyset$," whose probability is the order parameter $M$. Since the part of the argument is purely graph-theoretic, we shall state it in that general context. In the unoriented case this claim appears to be selfevident, until one tries to prove it. 

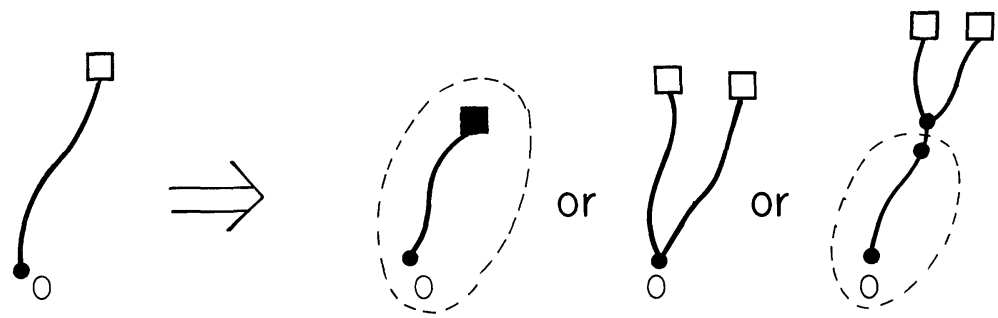

Fig. 2. The decomposition of the event " $C(0) \cap G \neq \emptyset$ " provided by Lemma 3.5. The solid square represents a single green site whereas the open squares represent subsets of the green set. The solid lines show connections made by paths of occupied bonds and the dashed line represents the "hypersurface" separating the cluster of the origin from the remainder of the lattice. Unless explicitly shown otherwise, all sites in the cluster are nongreen

Lemma 3.5. Let $\Gamma$ be a partially directed graph: a set of sites (vertices) and directed and undirected edges which link some of the pairs of sites.

If a site $z$ in $\Gamma$ is connected to at least one site belonging to a given subset $G$ of sites, then exactly one of the following three possibilities holds (see Fig. 2).

(1) The site $z$ is connected to exactly one site in $G$.

(2) The site $z$ is doubly connected to $G$ (in the sense defined above).

(3) There is an edge linking a pair of sites $\{x, y\}$ such that in the subgraph obtained by the removal of this edge from $\Gamma$,

(i) $z$ is connected to $x$,

(ii) $z$ is not connected to any sites in $G$, and

(iii) $y$ is doubly connected to $G$.

Proof. It is easily seen that (1), (2), and (3) are disjoint. Thus the heart of the matter is to show that if $z$ is connected to $G$ and the first two possibilities do not occur, then the third must occur. So we suppose that

(a) $z$ is connected to more than one site in $G$, and

(b) $z$ is not doubly connected to $G$.

Let $S$ be the set of all sites which are doubly connected to $G$. From our assumptions, we can make the following observations.

(A) $S$ does not include $z$. [Use (b).]

(B) $S$ is not empty. To see this, let us take any two self-avoiding (hereafter, s.a.) paths $\gamma_{1}$ and $\gamma_{2}$ from $z$ to $G$. Such paths exist by (a). The set of sites visited by both paths is nonempty since it includes $z$. The "last" vertex in this set - in the order of, say, $\gamma_{1}-$ is clearly in $S$.

(C) The site $z$ is connected to $S$. (This is another consequence of the preceding construction.)

(D) Any path from $z$ to $G$ must hit a site in $S$. Let us first comment that this statement is obvious in the undirected case where each edge connects both of the sites to which it is associated because every site of $G$ is also in $S$. For the more general case, an adaptation of the argument of (B) proves (D).

(E) Any two distinct sites $u$ and $v$ in $S$ are disjointly connected to $G$. To see this, let $\gamma_{1}$ and $\gamma_{2}$ be two s.a. and disjoint (meaning that they have no edges in common) paths from $u$ to $G$. Now let $\gamma_{3}$ be any s.a. path connecting $v$ to $G$. If $\gamma_{3}$ fails to 
intersect both $\gamma_{1}$ and $\gamma_{2}$ we are done, so suppose that it intersects at least one of them. Without loss of generality, we may assume that $\gamma_{3}$ intersects $\gamma_{2}$ before $\gamma_{1}$. Combining the first part of $\gamma_{3}$ with the latter portion of $\gamma_{2}$ yields a path from $v$ to $G$ which is disjoint from $\gamma_{1}$.

Let us now choose an s.a. path $\gamma$ from $z$ to $S$ which stops upon hitting $S$ for the first time, and let $b$ denote the last edge along this path. We claim that $b$ has the following property (from which it follows that $b$ is unique).

(F) Every path from $z$ to $S$ passes through $b$. To verify this, suppose that there exists some other s.a. path $\gamma^{\prime}$ connecting $z$ to $S$ which first hits $S$ through some other edge $b^{\prime}$. If the two paths intersect only at $z$ (and, possibly, their other endpoint) then $z$ is doubly connected to $G$ which contradicts assumption (b). But if $\gamma$ and $\gamma^{\prime}$ have another intersection point, then, by the construction used in (B), they must visit a site in $S$ before reaching their endpoints, which is also a contradiction.

Not only is $b$ a choke-point for all connections from $z$ to $S$, but also, by (D), every path from $z$ to $G$ passes through $b$. This statement shows that the third of the alternatives listed in the lemma does indeed occur if the first two do not.

Proof of Proposition 3.2. We will establish inequality (3.2) by first proving the related bound

$$
M_{L} \leqq \tilde{h} \chi_{L}+M_{L}^{2}+\beta Q M_{L} \frac{\partial M_{L}}{\partial \beta} \text {, }
$$

where $\tilde{x}=e^{x}-1(\cong x$, for small $x), Q=\sup _{b}\left\{\widetilde{\beta J_{b}^{(L)}} / \beta J_{b}^{(L)}\right\}$ and the supremum is over all oriented, and unoriented, bonds $b$ [see the comment after (3.3)]. We then show that $Q$ may be replaced by one and that $\tilde{h}$ may be replaced by $h$.

We begin applying Lemma 3.5 to the situation where $\Gamma$ is the set of sites and bonds in $\Lambda_{L}, z$ is the origin and $G$ is the green set. With (2.16) we find

$$
M_{L}=\operatorname{Prob}_{L}(C(0) \cap G \neq \emptyset)=\operatorname{Prob}_{L}\left(F_{1}\right)+\operatorname{Prob}_{L}\left(F_{2}\right)+\operatorname{Prob}_{L}\left(F_{3}\right),
$$

where $F_{1}, F_{2}$, and $F_{3}$ are the events corresponding to the three possibilities listed in Lemma 3.5.

Partitioning the event $F_{1}$ according to the number $n$ of sites in $C(0)$, and then computing the probability that exactly one of the $n$ sites is green yields

$$
\operatorname{Prob}_{L}\left(F_{1}\right)=\left(e^{h}-1\right) \sum_{n} P_{n}^{(L)} n e^{-n h}=\tilde{h} \chi_{L} .
$$

Application of the van den Berg-Kesten inequality to the event $F_{2}$ produces

$$
\operatorname{Prob}_{L}\left(F_{2}\right) \leqq\left[\operatorname{Prob}_{L}(C(0) \cap G \neq \emptyset)\right]^{2}=M_{L}^{2} .
$$

By the subadditivity of the probability measure,

$$
\begin{aligned}
& \operatorname{Prob}_{L}\left(F_{3}\right) \leqq \sum_{b} \operatorname{Prob}_{L}\left(n_{b}=1, x \in C(0), C^{\sim b}(0) \cap G=\emptyset,\right. \\
& y \text { is doubly connected to } G) .
\end{aligned}
$$

Here $b$ is either $(x, y)$ or $\{x, y\}$ and $C^{\sim b}(0)$ is the cluster of sites which remain connected to the origin even after $n_{b}$ is set to zero. 


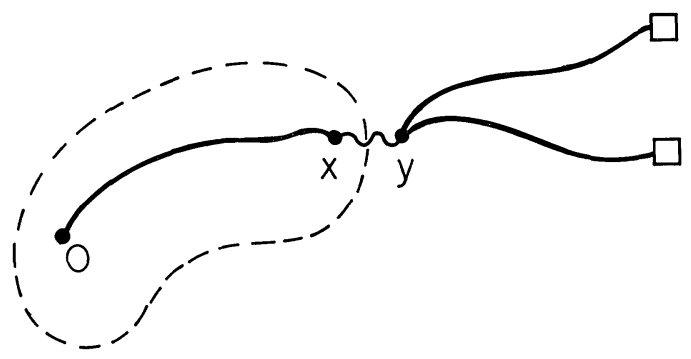

Fig. 3. The event that $n_{b}=1, x \in C(0), C^{\sim b}(0) \cap G=\emptyset$, and $y$ is doubly connected to $G$. The solid lines show connections made by chains of occupied bonds, the wiggly line is the occupied bond $b_{0}$, the two squares represent the two disjoint subsets of $G$ to which $y$ is connected, and the dashed line portrays the hypersurface which outlines $C^{\sim b}(0)$. Every bond crossing this surface which could connect the interior to the exterior (except for $b$ ) is unoccupied

The events in the right-hand side of (3.10) are depicted in Fig. 3, which is somewhat similar to Fig. 1. The remainder of the proof consists of relating the corresponding terms. The van den Berg-Kesten inequality allows the replacement of one of the connections from $y$ to $G$ with the probability that $y$ is connected to $G$, i.e. the order parameter. Some minor adjustments are then necessary to compensate for the fact that $b$ is occupied in Fig. 3 and unoccupied in Fig. 1.

The details of the above argument are as follows. Partitioning the events on the right-hand side of (3.10) according to $C^{\sim b}(0)$, and using the independence of the resulting events, we have that

$$
\begin{aligned}
\operatorname{Prob}_{L}\left(F_{3}\right) \leqq & \sum_{x, y \in A_{L}} \sum_{A \subset \Lambda_{L}: x \in A} \operatorname{Prob}_{L}\left(n_{\{x, y\}}=1\right) \\
& \times \operatorname{Prob}_{L}\left(A=C^{\sim\{x, y\}}(0), A \cap G=\emptyset\right) \\
& \times \operatorname{Prob}_{L}\left(y \text { is doubly connected to } G \text { in } \Lambda_{L} \backslash A\right) \\
& +(\text { a similar sum with }\{x, y\} \text { replaced by }(x, y)) .
\end{aligned}
$$

The combination of the van den Berg-Kesten inequality and the observation that the order parameter in $\Lambda_{L}$ is larger than the order parameter in $\Lambda_{L} \backslash A$ yields

$\operatorname{Prob}_{L}\left(y\right.$ is doubly connected to $G$ in $\left.\Lambda_{L} \backslash A\right) \leqq M_{L} \operatorname{Prob}_{L}\left(C_{A_{L} \backslash A}(y) \cap G \neq \emptyset\right)$.

Inserting this bound in the right-hand side of the preceding inequality and comparing $\operatorname{Prob}_{L}\left(n_{b}=0\right)$ with $\operatorname{Prob}_{L}\left(n_{b}=1\right)$, we obtain

$$
\begin{aligned}
\operatorname{Prob}_{L}\left(F_{3}\right) \leqq & M_{L} \sum_{x, y \in \Lambda_{L}} \operatorname{Prob}_{L}(x \in C(0), C(0) \cap G=\emptyset, C(y) \cap G \neq \emptyset) \\
& \times\left(\widetilde{\beta J_{x, y}^{(L)}}+\widetilde{\beta J_{x \rightarrow y}^{(L)}}\right) \\
\leqq & \beta Q M_{L} \sum_{x, y \in \Lambda_{L}} \operatorname{Prob}_{L}(x \in C(0), C(0) \cap G=\emptyset, C(y) \cap G \neq \emptyset) \\
& \times\left(J_{x, y}^{(L)}+J_{x \rightarrow y}^{(L)}\right),
\end{aligned}
$$

where $Q=\sup _{b}\left\{\widetilde{\beta J_{b}^{(L)}} / \beta J_{b}^{(L)}\right\}$. With (3.4) this becomes

$$
\operatorname{Prob}_{L}\left(F_{3}\right) \leqq \beta Q M_{L} \frac{\partial M_{L}}{\partial \beta} .
$$


The three terms appearing in the decomposition (3.7) of $M_{L}$ have been computed and bounded in (3.8), (3.9), and (3.11). Together these relations yield (3.6).

To obtain (3.2) we need to replace the $Q$ and $\tilde{h}$ in (3.6) with $1(<Q)$ and $h(<\tilde{h})$. So that the same argument may be used to justify each of these substitutions we first show how the ghost field $h$ may be put on more of an equal footing with the bond density parameter $\beta$. We append to the lattice $\mathbb{L}$ a single green site $g$ (the point at infinity) which is connected by "ghost bonds" to every site $x$ in $\mathbb{L}$. The site variable $m_{x}$ is now interpreted as the bond variable for the ghost bond $\{x, g\}$, i.e.,

$$
\operatorname{Prob}\left(n_{\{x, g\}}=1\right)=\operatorname{Prob}\left(m_{x}=1\right)=1-e^{-h} .
$$

If we replace " $x \in G$ " by " $x$ is directly connected to $g$ by the occupied bond $\{x, g\}$ " and understand that $|C(0)|$ denotes the cardinality of lattice sites excluding $g$ to which the origin is connected, then none of our preceding work is modified. For the rest of this argument we will work from the ghost bond rather than the green site point of view.

We now replace the partially ordered percolation model by a refinement which has a different set of bonds, but exactly the same connectivity functions. The new system is obtained by splitting each bond $b$ into $n$ different bonds which connect the same pair of sites (and have the same orientation) as $b$. The couplings for the new bonds are chosen to be $J_{b} / n$ (or $h / n$ for the new ghost bonds). The original model may still be realized within the new one by regarding the bond $b$ as occupied whenever at least one of the bonds in the collection obtained by splitting $b$ is occupied. It is easy to see that the function $M_{L}(\beta, h)$ - and its $\beta$ and $h$ derivatives for the refined model are the same as that of the original system.

Repeating the argument which proved (3.6), we find that the terms on the righthand side of (3.7) can be evaluated or bounded for the refined system as follows:

$$
\begin{aligned}
& \operatorname{Prob}_{L}\left(F_{1}\right)=n \overline{\left(\frac{h}{n}\right)} \chi_{L}, \\
& \operatorname{Prob}_{L}\left(F_{2}\right) \leqq M_{L}^{2} \quad \text { (as before), }
\end{aligned}
$$

and

$$
\operatorname{Prob}_{L}\left(F_{3}\right) \leqq n Q_{n} \beta M_{L} \frac{\partial M_{L}}{\partial \beta},
$$

where $\left.Q_{n}=\sup _{b}\left\{\overline{\left(\beta J_{b}^{(L)}\right.} / n\right) / \beta J_{b}^{(L)}\right\}$. The prefactor $n$ in (3.12) arises from the fact that there are $n$ choices at each site for the single ghost bond connecting $C(0)$ to $g$. Similarly, the prefactor $n$ in (3.14) is due to there being $n$ choices in the refined system for the "pivotal" lattice bond produced by Lemma 3.5.

Combining (3.7) with (3.12)-(3.14) and observing that

$$
n \overline{\left(\frac{h}{n}\right)} \underset{n \rightarrow \infty}{\longrightarrow} h,
$$

and

$$
n Q_{n} \underset{n \rightarrow \infty}{\longrightarrow} 1
$$

allows us to conclude that (3.2) holds. 


\section{A Lower Bound on $M\left(\beta_{T}, h\right)$}

In this section we prove Theorem 1.2 for the translation invariant partially oriented percolation models introduced in Sect. 2.1. That is, we show

$$
M\left(\beta_{T}, h\right) \geqq \text { const } h^{1 / 2} .
$$

The first step in obtaining the bound (4.1) is to combine the two partial differential inequalities (3.1) and (3.2) to obtain a single ordinary differential inequality for $M_{L}$. The substitution of (3.1) into (3.2) yields

$$
M_{L} \leqq h \frac{\partial M_{L}}{\partial h}+M_{L}^{2}+\beta M_{L} \frac{\partial M_{L}}{\partial \beta} \leqq h \frac{\partial M_{L}}{\partial h}+M_{L}^{2}+\beta|J| M_{L}^{2} \frac{\partial M_{L}}{\partial h} .
$$

Before proceeding to the proof of (4.1), let us pause to examine the different implications, for $\beta<\beta_{T}$ and $\beta=\beta_{T}$, of the infinite volume limit (see Proposition 2.3) of (4.2):

$$
M \leqq h \frac{\partial M}{\partial h}+M^{2}+\beta|J| M^{2} \frac{\partial M}{\partial h} .
$$

Remark. By weakening (3.2) one step further, we obtain

$$
M \leqq h \frac{\partial M}{\partial h}+M^{2}+\beta|J| \frac{M^{3}}{h} .
$$

This inequality follows from (4.3) by the application of the bound

$$
\frac{\partial M}{\partial h}(\beta, h) \leqq \frac{M(\beta, h)-M(\beta, 0)}{h} \leqq \frac{M(\beta, h)}{h},
$$

which is implied by the concavity in $h$ of $M(\beta, h)$. It is instructive to compare (4.4) with the inequality (1.21) which was previously derived by Fröhlich and Sokal [15] for ferromagnetic spin systems. Inequality (1.21) implies the critical exponent bound $\delta \geqq 3$ for those models.

To develop intuition for our treatment of (4.3) let us suppose that, for a given $\beta, M(\beta, h)$ displays strict power law behavior in the sense that $M=c h^{s}$. Then the operator $h \frac{\partial}{\partial h}$ acts as multiplication by $s$ when applied to $M$. For $\beta<\beta_{T}, M(\beta, 0)=0$, and $\chi(\beta, 0)<\infty$; hence $s=1$. In this case, (4.3) is of little interest in the $h \searrow 0$ limit - its left-hand side is entirely cancelled by the first term on the right. However, for $\beta=\beta_{T}, M$ is expected to have $s=\frac{1}{\delta}<1$. Then $h \frac{\partial M}{\partial h}=\frac{1}{\delta} M$ and this term can no longer completely cancel the left-hand side of (4.3). So under the strict power law assumption, one would have

$$
\left(1-\frac{1}{\delta}\right) M-M^{2} \leqq \beta_{T}|J| \frac{M^{3}}{h}
$$

from (4.4). The above inequality implies that $\frac{M^{2}}{h} \geqq$ const $>0$, i.e., $\delta \geqq 2$. Note that 
for percolation models $\delta=2$ in the mean field approximation, and on a Bethe lattice.

We now offer a rigorous argument which leads us from the differential inequality (4.3) to the bound (4.1) without having to make any additional assumptions, such as power law behavior. To make our analysis applicable also to other models (see Sects. 7 and 8), we present the next result in greater generality than is actually required here.

Lemma 4.1. Let $M(h)$ be an increasing differentiable function of $h$ for $h>0$ obeying

$$
\begin{aligned}
& M \searrow 0 \quad \text { as } \quad h \searrow 0, \\
& \frac{M}{h} \rightarrow \infty \quad \text { as } \quad h \searrow 0,
\end{aligned}
$$

and

$$
M \leqq h \frac{d M}{d h}+M f(M)+a M^{1+\theta} \frac{d M}{d h},
$$

where $a, \theta \in(0, \infty)$ and $f$ satisfies

$$
\begin{gathered}
0 \leqq f(M)<1, \\
\text { ii) } \quad f(M) \rightarrow 0 \text { as } M \searrow 0,
\end{gathered}
$$

and

$$
\int_{0}^{1} \frac{f(M)}{M} d M<\infty
$$

Then when $h$ is small,

$$
M \geqq \text { const } h^{1 / 1+\theta}
$$

with a positive constant.

Proof. Since $M$ is increasing and differentiable it has a differentiable inverse $h(M)$. Rewrite (4.8) as

$$
\frac{d h}{d M}-\frac{h}{M(1-f(M))} \leqq \frac{a M^{\theta}}{1-f(M)}
$$

and multiply this inequality by the integrating factor $\frac{1}{\mu}$, where

$$
\mu(M)=\exp \left\{-\int_{M}^{1 / 2} \frac{d x}{x(1-f(x))}\right\} .
$$

The resulting expression is

$$
\frac{d}{d M}\left(\frac{h}{\mu}\right) \leqq \frac{a M^{\theta}}{(1-f(M)) \mu(M)} .
$$



since

Under assumption (4.11), the quantity $\mu(M)$ behaves linearly in $M$ as $M \searrow 0$

$$
\frac{M}{\mu(M)}=\frac{1}{2} \exp \left\{\int_{M}^{1 / 2} \frac{d x}{x}\left[\frac{1}{1-f(x)}-1\right]\right\} \underset{M \rightarrow 0}{\longrightarrow} \exp \left\{\int_{0}^{1 / 2} \frac{f(x) d x}{x(1-f(x))}\right\}<\infty .
$$

Thus, by (4.7), $\frac{h}{\mu} \rightarrow 0$ as $M \searrow 0$.

The integration of (4.13) from 0 to $M$ yields

$$
\frac{h(M)}{\mu(M)} \leqq a \int_{0}^{M} \frac{x^{\theta} d x}{(1-f(x) \mu(x)},
$$

from which we obtain

$$
h \leqq \text { const } M^{1+\theta}
$$

and hence (4.12).

Theorem 4.2. For every translation invariant bond percolation model on $\mathbb{Z}^{d}$, the order parameter obeys the following bound (4.1):

$$
M\left(\beta_{T}, h\right) \geqq \text { const } h^{1 / 2}
$$

for small $h$. In particular, if $M\left(\beta_{T}, 0\right)=0$, then the critical exponent $\delta$ defined by (1.12) is bounded from below:

$$
\delta \geqq 2 .
$$

Proof. If $M$ is discontinuous at $\left(\beta=\beta_{T}, h=0\right)$, i.e., $M\left(\beta_{T}, 0\right)>0$, there is nothing to prove. So suppose

$$
M\left(\beta_{T}, 0\right)=0 .
$$

We want to apply Lemma 4.1 with $M(h)=M\left(\beta_{T}, h\right)$. By Lemma $2.4, M$ is increasing and differentiable. Condition 1) of Lemma 4.1 is met by the assumption (4.16) and the continuity of $M(\beta, h)$ in $h$. From (4.16), (2.8) and the fact that $\chi_{\text {total }}(\beta)$ diverges as $\beta \uparrow \beta_{T}[7,8]$, we see that $\chi$ is infinite at $\left(\beta=\beta_{T}, h=0\right)$. Together with (4.5), this observation shows that $M\left(\beta_{T}, h\right) / h$ diverges as $h \searrow 0$. Let us emphasize that it it precisely this point that the criticality of $\beta_{T}$ has entered our argument. Condition 3 ) of the lemma is satisfied by (4.3) with $f(M)=M, \theta=1$, and $a=\beta_{T}|J|$. A more explicit version of (4.14) can be obtained in this case:

$$
h \leqq \beta_{T}|J| \frac{M^{2}}{1-M} .
$$

\section{Equality of $\beta_{T}$ and $\beta_{H}$}

In this section we prove the general result on the equality of the two critical points defined in the introduction for homogeneous partially oriented bond percolation models. 
Dividing both sides of (3.2) by $M_{L}$, which is positive when $h$ is, we obtain an inequality which provides a lower bound on $\frac{\partial M_{L}}{\partial \beta}$ :

$$
1 \leqq \frac{h \chi_{L}}{M_{L}}+M_{L}+\beta \frac{\partial M_{L}}{\partial \beta} \quad(h>0) .
$$

Let us now discuss what becomes of the first term on the right-hand side of (5.1) after taking the infinite volume limit and then the $h \searrow 0$ limit. If $M(\beta, 0)=0$, then $\lim _{h \searrow 0} \frac{M(\beta, h)}{h}=\chi(\beta, h)$, since $\chi$ is continuous from the right at $h=0$. However, when $M(\beta, 0)>0$, the leftmost inequality in (4.5) implies that

$$
0 \leqq \frac{h \chi(\beta, h)}{M(\beta, h)} \leqq 1-\frac{M(\beta, 0)}{M(\beta, h)} .
$$

Thus we have

$$
\lim _{h \searrow 0} \frac{h \chi(\beta, h)}{M(\beta, h)}=\left\{\begin{array}{ll}
1 & \beta<\beta_{T}, \\
0 & \beta>\beta_{H}
\end{array} .\right.
$$

Were $\beta_{H}$ strictly greater than $\beta_{T}$ we would discover in the intermediate regime that $M(\beta, 0)=0$ and (by Theorem 4.2 and Lemma 2.5$) M(\beta, h) \geqq c h^{1 / 2}$. It would be natural then to assume the existence of the following limits:

$$
\lim _{h \searrow 0} \frac{h \chi(\beta, h)}{M(\beta, h)}=\lim _{h \searrow 0} \frac{\partial \ln M}{\partial \ln h} \equiv \frac{1}{\delta(\beta)} \quad\left(\beta_{T} \leqq \beta<\beta_{H}\right) .
$$

In this case, Lemma 2.5 and Theorem 4.2 imply

$$
\frac{1}{\delta(\beta)} \leqq \frac{1}{\delta\left(\beta_{T}\right)}=\frac{1}{2} .
$$

Using (5.3) to substitute (5.4) into the formal expression obtained by taking the limits $L \rightarrow \infty$ and $h \searrow 0$ in (5.1) would yield

$$
\frac{\partial M}{\partial \beta}(\beta, 0) \geqq \frac{1}{2}-M(\beta, 0) \quad\left(\beta_{T} \leqq \beta<\beta_{H}\right) .
$$

However, integration of (5.5) shows that $M(\beta, 0)$ becomes positive as soon as $\beta$ is larger than $\beta_{T}$. Hence $\beta_{T}$ and $\beta_{H}$ coincide and there is no intermediate region after all.

The actual proof presented below was designed to arrive at (5.5) circumventing all the assumptions made in the above heuristic argument.

Before presenting the proof of the main result let us comment that, for $\beta>\beta_{H}$, the combination of (5.1) and (5.2) leads us to the inequality of Chayes and Chayes [14]:

$$
\beta \frac{\partial M}{\partial \beta}(\beta, 0) \geqq 1-M(\beta, 0) .
$$


Here the derivative is to be interpreted in the distributional sense. The integration of (5.6) (which is justified by the argument of Chayes and Chayes, or by the method used in the proof of Theorem 5.1) shows that

$$
M(\beta, 0)-M\left(\beta_{H}, 0\right) \geqq\left[1-M\left(\beta_{H}, 0\right)\right]\left(\frac{\beta-\beta_{H}}{\beta}\right)
$$

for $\beta \geqq \beta_{H}$. This leads to the mean field bound

$$
\hat{\beta} \leqq 1
$$

for the critical exponent $\widehat{\beta}$ defined in (1.17).

For a complete proof of the above statements we present here a useful lemma which shows the consequences of a more general inequality than (3.2). (In this generality, the analysis is applicable also to Ising models [22].)

Lemma 5.1. Let $\left\{M_{L}(\beta, h)\right\}$ be a sequence of positive functions defined for $\beta, h>0$, increasing and differentiable in both $\beta$ and $h$, and converging as $L \rightarrow \infty$ to the function $M(\beta, h)$ which is extended to $h=0$ so as to be continuous in $h$ there. Suppose that the functions $M_{L}$ obey

$$
M_{L} \leqq h \frac{\partial M_{L}}{\partial h}+M_{L} f\left(M_{L}\right)+a_{1} M^{\theta} \frac{\partial M_{L}}{\partial \beta},
$$

and

$$
\frac{\partial M_{L}}{\partial \beta} \leqq a_{2} M_{L} \frac{\partial M_{L}}{\partial h},
$$

where $a_{1}, a_{2}, \theta \in(0, \infty)$ and $f$ is a continuous function satisfying conditions (4.9)-(4.11) of Lemma 4.1. If there exists a $\beta_{0}$ for which

$$
\frac{M\left(\beta_{0}, h\right)}{h} \rightarrow \infty
$$

as $h \searrow 0$, then for each $\beta>\beta_{0}$,

$$
M(\beta, 0) \geqq \operatorname{const}\left(\beta-\beta_{0}\right)^{1 / \theta}
$$

with a positive constant.

For the sake of concreteness, let us mention that the bound which we obtain is

$$
M^{\theta}\left(\beta_{2}, 0\right)-M^{\theta}\left(\beta_{1}, 0\right) \geqq \frac{\theta}{a_{1}}\left[1-\widehat{f}\left(M\left(\beta_{1}, 0\right), M\left(\beta_{2}, 0\right)\right)\right]\left(\beta_{2}-\beta_{1}\right)
$$

for all $\beta_{2} \geqq \beta_{1} \geqq \beta_{0}$, where $\hat{f}(x, y)=\sup \{f(z) \mid x \leqq z \leqq y\}$. With $\beta_{1}=\beta_{0}$, this bound proves (5.12). With $\beta_{2}-\beta_{1}$ infinitesimal, (5.13) shows that $\frac{\partial M^{\theta}}{\partial \beta} \geqq \frac{\theta}{a_{1}}[1-f(M)]$ in the distributional sense, just as suggested by the above heuristic argument.

Proof. Our starting point is the inequality (5.9) which is rewritten as

$$
1 \leqq \frac{\partial \ln M_{L}}{\partial \ln h}+f\left(M_{L}\right)+\frac{a_{1}}{\theta} \frac{\partial M_{L}^{\theta}}{\partial \beta} .
$$


We integrate (5.14) over the rectangle $\mathscr{R}=\left[\beta_{0}, \beta\right] \times\left[h_{1}, h_{2}\right]$, where $\beta_{0}<\beta$ and $0<h_{1}<h_{2}$, with the measure $\frac{1}{h} d h d \beta=d \ln h d \beta$. Observe that by the monotonicity properties of the functions $M_{L}$

$$
\min \left\{M_{L}\left(\beta^{\prime}, h\right) \mid\left(\beta^{\prime}, h\right) \in \mathscr{R}\right\}=M_{L}\left(\beta_{0}, h_{1}\right)
$$

and

$$
\max \left\{M_{L}\left(\beta^{\prime}, h\right) \mid\left(\beta^{\prime}, h\right) \in \mathscr{R}\right\}=M_{L}\left(\beta, h_{2}\right) .
$$

By our choice of measure, one of the two iterated integrals of each of the derivative terms in (5.14) can be integrated exactly. The remaining integrations are performed by making appropriate use of the previously mentioned bounds on $M_{L}$. In the $L \rightarrow \infty$ limit, one obtains

$$
\left(\beta-\beta_{0}\right) \ln \frac{h_{2}}{h_{1}} \leqq\left(\beta-\beta_{0}\right) \ln \frac{M_{2}}{M_{1}}+\left(\beta-\beta_{0}\right) \ln \frac{h_{2}}{h_{1}} \hat{f}\left(M_{1}, M_{2}\right)+\frac{a_{1}}{\theta}\left[M_{2}^{\theta}-M_{1}^{\theta}\right] \ln \frac{h_{2}}{h_{1}},
$$

where $M_{1}=M\left(\beta_{0}, h_{1}\right)$ and $M_{2}=M\left(\beta, h_{2}\right)$.

Next keep $h_{2}>0$ fixed and let $h_{1} \searrow 0$. The singular factor $\ln \frac{h_{2}}{h_{1}}$ appears explicitly in three of the four terms in (5.15). To handle the remaining term we consider two cases: either $M\left(\beta_{0}, 0\right)=0$ or $M\left(\beta_{0}, 0\right)>0$. In the first case, Lemma 4.1 implies

$$
\ln \frac{M_{2}}{M_{1}} \leqq \ln \frac{M_{2}}{\operatorname{const~} h_{2}^{1 / 1+\theta}}+\frac{1}{1+\theta} \ln \frac{h_{2}}{h_{1}},
$$

and, in the second case,

$$
\ln \frac{M_{2}}{M_{1}} \leqq \ln \frac{M_{2}}{\text { const }}
$$

Thus dividing both sides of (5.15) by $\ln \frac{h_{2}}{h_{1}}$, using the appropriate bound on $\ln \frac{M_{2}}{M_{1}}$, and taking the limit, we obtain

$$
M_{2}^{\theta}-M^{\theta}\left(\beta_{0}, 0\right) \geqq \begin{cases}\frac{\theta}{a_{1}}\left[1-\frac{1}{1+\theta}-\hat{f}\left(M\left(\beta_{0}, 0\right), M_{2}\right)\right]\left(\beta-\beta_{0}\right), & M\left(\beta_{0}, 0\right)=0, \\ \frac{\theta}{a_{1}}\left[1-\hat{f}\left(M\left(\beta_{0}, 0\right), M_{2}\right)\right]\left(\beta-\beta_{0}\right), & M\left(\beta_{0}, 0\right) \geqq 0 .\end{cases}
$$

In the limit $h_{2} \searrow 0, M_{2}$ is replaced in (5.16) with $M(\beta, 0)$. The resulting bound proves the claim made in (5.12).

Regarding the inequality (5.13), let us note that the assumption made on $\beta_{0}$ in Lemma 5.1 is satisfied also for each $\beta_{1}>\beta_{0}$. A posteriori, we now know that in this case the second of the alternatives in (5.16) applies. Hence (5.16) proves the bound (5.13) for all $\beta_{2} \geqq \beta_{1}>\beta_{0}$. The extension to the case $\beta_{1}=\beta_{0}$ follows by simple considerations. 
Theorem 5.2. For a translation invariant partially oriented percolation model on $\mathbb{Z}^{d}$,

$$
\beta_{T}=\beta_{H} \quad\left(\equiv \beta_{c}\right) .
$$

Proof. The content of the theorem is that $M(\beta, 0)$ becomes positive as soon as $\beta>\beta_{T}$, where $\beta_{T}$ is defined as in (1.5). This result follows by a direct application of the preceding lemma taking $\beta_{0}=\beta_{T}$. The hypotheses on the functions $M_{L}$ are shown to be satisfied by Lemmas $2.3-2.5$ and by Propositions 3.1 and 3.2, with $\theta=1$. The ratio $\frac{M\left(\beta_{T}, h\right)}{h}$ diverges as $h \searrow 0$ by the argument given in the proof of Theorem 4.2. The explicit bound our methods yield for (bond) percolation is the inequality (5.7).

\section{Extrapolation Principles}

In this section we shall present some additional information on the critical behavior in percolation models which follows, by known arguments, from inequality (3.1). The main results are the critical exponent bound

$$
\widehat{\beta}(\delta-1) \geqq 1,
$$

and a statement which proves one of the general predictions of the scaling hypothesis (Proposition 6.1).

As was already stated in the introduction, (3.1) is identical to a relation obeyed by the derivatives of the order parameter (i.e., magnetization) in ferromagnetic Ising models. In that context, it was pointed out by Newman [11], that (3.1) leads to (6.1). Newman's observation was expanded in [12] to two extrapolation principles, which are now made applicable to percolation.

The hypotheses under which these extrapolation principles were derived are the following properties of the order parameter $M$ and its finite volume approximations.

1. $M(\beta, h)$ is continuous in $h$ for $h \geqq 0$.

2. For $h>0, M(\beta, h)$ is the pointwise limit of the functions $M_{L}(\beta, h)$ which are differentiable in $h$.

3. The functions $M_{L}$ satisfy

$$
0 \leqq \frac{\partial M_{L}}{\partial \beta} \leqq|J| M_{L} \frac{\partial M_{L}}{\partial h}
$$

and

$$
0 \leqq \frac{\partial M_{L}}{\partial h} .
$$

Observe that from (3.1), (2.18), and Lemmas 2.4 and 2.5, these conditions are satisfied for the percolation models considered in this paper. The most important of these properties is the inequality (3.1).

Perhaps the simplest way of viewing (3.1) to interpret it as providing a bound on the slopes of curves in the $\beta-h$ plane on which the order parameter is constant. 
Since $M$ is an increasing function of $h$ for fixed $\beta$, the relation $M(\beta, h)=m$ can be inverted to define the function $h(\beta, m)$. By Lemmas 2.4 and 2.5 , for each fixed value of $m$, the curve $h=h(\beta, m)$ in the $\beta-h$ plane has negative slope. Inequality (3.1) tells us that the slope of such a curve of constant $M$ is bounded, in absolute value, by $|J| m:$

$$
0 \leqq-\frac{\partial h}{\partial \beta}(\beta, m) \leqq|J| m .
$$

This bound on the slopes makes it possible to compare the critical behavior of the order parameter on different lines of approach to the critical point $\left(\beta=\beta_{c}, h=0\right)$.

In this section we will only state the extrapolation principles. Readers who are interested in the proofs are directed to [12].

Proposition 6.1. Suppose

$$
M\left(\beta_{c}+t, h\right) \geqq c h^{\alpha}|\ln h|^{\omega}(1+O(h))
$$

along a ray $t=a h, h \geqq 0$ with $c>0,0<\alpha<1$, and $\omega \geqq 0$. Then a similar inequality with the same $c, \alpha$, and $\omega$ is asymptotically true for any other ray $t=b h, h \geqq 0$. The same principle applies to upper bounds on $M$.

Proposition 6.2. (i) Suppose

$$
M\left(\beta_{c}+t, 0\right) \geqq c t^{\lambda}(1+O(t))
$$

in the region $R_{2}=\left\{(\beta, h) \mid \beta \geqq \beta_{c}, h=0\right\}$ with $c, \lambda>0$. Then along any ray $t=a h, h \geqq 0$,

$$
M\left(\beta_{c}+t, h\right) \geqq\left(|J| c^{1 / \lambda}\right)^{\lambda / 1+\lambda} h^{\lambda / 1+\lambda}(1+O(h)) .
$$

(ii) Suppose

$$
M\left(\beta_{c}+t, h\right) \leqq c h^{\alpha}|\ln h|^{\omega}(1+O(h))
$$

along a ray $t=a h, h \geqq 0$, with $c>0,0<\alpha<1$, and $\omega \geqq 0$. Then, in the region $R_{2}$,

$$
M \leqq\left(|J| c^{1 / \alpha}\right)^{\alpha / 1-\alpha} t^{\alpha / 1-\alpha}|\ln (|J| M t)|^{\omega / 1-\alpha}(1+O(t)),
$$

where $M=M\left(\beta_{c}+t, 0\right)$.

Proposition 6.2(i) implies the following critical exponent inequality which is satisfied also in Ising models.

Corollary 6.3. The critical exponents $\delta$ and $\widehat{\beta}$ as defined in (1.12) and (1.17) obey the relation (6.1):

$$
\hat{\beta}(\delta-1) \geqq 1 .
$$

The above inequality is saturated in the mean field approximation - for percolation, as well as for Ising models.

Note that by the results of the preceding sections, (6.4) and (6.5) are satisfied with $\lambda=1(\geqq \widehat{\beta})[14]$. However, before one knows that $\beta_{T}=\beta_{H}$, the combination of Corollary 6.3 and (5.8) only proves [see (5.3)]

$$
\delta\left(\beta_{H}\right) \geqq 2,
$$

which is a weaker statement than (4.15) since [by (5.4)] $\delta\left(\beta_{H}\right) \geqq \delta\left(\beta_{T}\right)$. 


\section{Site Percolation}

Heretofore we have proven Theorems 1.1 and 1.2 only for partially oriented bond percolation models on $\mathbb{Z}^{d}$. In this section we extend the results which were derived for bond percolation models to translation invariant site percolation systems on $\mathbb{Z}^{d}$. In these models, the sites are each independently occupied with probability $p$.

In the standard nearest neighbor models, the sites $x$ and $y$ are directly connected if both are occupied and $|x-y|=1$. More generally, we let $\mathscr{N}$ be a given finite set of "neighbors" of the origin. The neighbors of the site $x$ are the translated neighbors of the origin: $\mathscr{N}+x$. So that the unoriented and oriented cases may be treated simultaneously, the set $\mathscr{N}$ is not required to be symmetric about the origin. We say that $x$ is connected to $y$ if there exists a sequence of occupied sites

$$
z_{1}=x, \quad z_{2}, \ldots, z_{n}=y \quad \text { with } \quad z_{i+1} \in \mathscr{N}+z_{i} .
$$

The cluster of $x$ could be defined as above to be the collection of all sites to which $x$ is connected. We denote this cluster by $c(x)$ as we will prefer to work with a different notion of cluster (called the augmented cluster in [8]):

$$
C(x)=\{x\} \cup \bigcup_{y \in \mathcal{H}+x} c(y) .
$$

[Note that if $x$ is occupied, $C(x)$ and $c(x)$ coincide.] With this new definition of cluster, the quantities $P_{n}, M$, and $\chi$ are defined by (1.4), (1.9), and (2.7) with $\beta$ replaced by $p$. The definitions of the critical probabilities $p_{H}$ and $p_{T}$ are still given by (1.5).

The results of Sect. 2 can be directly translated into results for site percolation by replacing $\beta$ with $p$ and the bond occupation variable $n_{b}$ with a site occupation variable $n_{x}\left(\operatorname{Prob}\left(n_{x}=1\right)=p\right)$. The finite volume models are determined by periodically continuing the neighbor sets of sites near the boundary of $\Lambda_{L}$.

We now state and briefly sketch the results which are the site percolation analogues of Propositions 3.1 and 3.2.

Proposition 7.1. For a homogeneous independent site percolation model on $\mathbb{Z}^{d}$,

$$
\frac{\partial M_{L}}{\partial p} \leqq \frac{|\mathcal{N}|}{1-p} M_{L} \chi_{L}
$$

Proposition 7.2. For the same models as above,

$$
M_{L} \leqq h \chi_{L}+M_{L}^{2}+p M_{L} \frac{\partial M_{L}}{\partial p} .
$$

Sketch of Proof of Proposition 7.1. By Russo's formula and the site percolation version of (2.16),

$$
\begin{aligned}
\frac{\partial M_{L}}{\partial p} & =\frac{\partial}{\partial p} \operatorname{Prob}_{L}(C(0) \cap G \neq \emptyset) \\
& =\frac{1}{1-p} \sum_{x \in \Lambda_{L}} \operatorname{Prob}_{L}(x \text { is vacant, } x \text { is pivotal for “ } C(0) \cap G \neq \emptyset \text { "). }
\end{aligned}
$$

It is to be understood that the pivotality of $x$ in (7.3) refers to its occupation status and not to its color. 
Following the argument presented in the proof of Proposition 3.1, (7.3) can be rewritten as

$$
\begin{aligned}
\frac{\partial M_{L}}{\partial p}= & \frac{1}{1-p} \sum_{x \in A_{L}} \sum_{A \subset A_{L}} \operatorname{Prob}_{L}(A=C(0), A \cap G=\emptyset, \\
& x \text { is a vacant neighbor of some site in } A) \\
& \times \operatorname{Prob}_{L}\left(C_{A_{L} \backslash A}(x) \cap G \neq \emptyset\right) \\
\leqq & \frac{1}{1-p} M_{L} \sum_{x \in \Lambda_{L}} \operatorname{Prob}_{L}(C(0) \cap G=\emptyset, \\
& x \text { is a vacant neighbor of some site in } C(0)) .
\end{aligned}
$$

Inequality (7.1) is obtained by applying the site version of (2.17) to the bound above since

$$
\begin{aligned}
& \sum_{x \in \Lambda_{L}} \operatorname{Prob}_{L}(C(0) \cap G=\emptyset, x \text { is a vacant neighbor of some site in } C(0)) \\
& \quad \leqq|\mathcal{N}| \sum_{x \in \Lambda_{L}} \operatorname{Prob}_{L}(x \in C(0), C(0) \cap G=\emptyset) . \quad
\end{aligned}
$$

Sketch of Proof of Proposition 7.2. Use Lemma 3.5 to decompose the event “ $C(0) \cap G \neq \emptyset$ " into the following three events.

$F_{1}: C(0)$ contains a single green site.

$F_{2}$ : The origin is doubly connected to $G$. By doubly connected we mean that, in the configuration obtained by requiring that the origin be occupied, there exist two distinct green sites $g$ and $g^{\prime}$ and a pair of disjoint (away from the origin) paths of sites (each site of which is an occupied neighbor of the preceding site) connecting the origin to $g$ and $g^{\prime}$.

$F_{3}$ : There exists a site $x$ such that

i) $x \in C(0)$,

ii) $C(0) \cap G=\emptyset$ in the configuration obtained by forcing the site $x$ to be vacant, and

iii) $x$ is doubly connected to $G$.

Thus, as before, we have

$$
\begin{gathered}
M_{L}=\operatorname{Prob}_{L}\left(F_{1}\right)+\operatorname{Prob}_{L}\left(F_{2}\right)+\operatorname{Prob}_{L}\left(F_{3}\right), \\
\operatorname{Prob}\left(F_{1}\right)=\tilde{h} \chi_{L},
\end{gathered}
$$

and

$$
\operatorname{Prob}\left(F_{2}\right) \leqq M_{L}^{2} \text {. }
$$

By the subadditivity of $\operatorname{Prob}_{L}$,

$$
\operatorname{Prob}_{L}\left(F_{3}\right) \leqq \sum_{x \in \Lambda_{L}} \operatorname{Prob}_{L}\left(x \in C(0), C^{\sim x}(0) \cap G=\emptyset, x \text { is doubly connected to } G\right),
$$

where $C^{\sim x}(0)$ is $C(0)$ in the configuration obtained by forcing the site $x$ to be vacant. We argue as in Proposition 3.2 to obtain

$$
\begin{aligned}
\operatorname{Prob}_{L}\left(F_{3}\right) \leqq & \sum_{x \in \Lambda_{L}} \sum_{A \subset A_{L}} I[x \text { is a neighbor of some site in } A] \\
& \times \operatorname{Prob}_{L}(x \text { is occupied }) \operatorname{Prob}_{L}\left(A=C^{\sim x}(0), A \cap G=\emptyset\right) \\
& \times \operatorname{Prob}_{L}\left(x \text { is doubly connected to } G \text { in } \Lambda_{L} \backslash A\right) .
\end{aligned}
$$


Applying the van den Berg-Kesten inequality, comparing $p=\operatorname{Prob}_{L}\left(x\right.$ is occupied) with $1-p=\operatorname{Prob}_{L}(x$ is vacant) and finally using (7.3), gives

$$
\begin{aligned}
\operatorname{Prob}_{L}\left(F_{3}\right) \leqq & p M_{L} \frac{1}{1-p} \sum_{x \in \Lambda_{L}} \sum_{A \subset \Lambda_{L}} \operatorname{Prob}_{L}(x \text { is a vacant neighbor } \\
& \text { of some site in } \left.A, A=C^{\sim x}(0), A \cap G=\emptyset\right) \operatorname{Prob}_{L}\left(C_{A_{L} \backslash A}(x) \cap G \neq \emptyset\right) \\
= & p M_{L} \frac{1}{1-p} \sum_{x \in \Lambda_{L}} \operatorname{Prob}_{L}(x \text { is vacant, } x \text { is pivotal for " } C(0) \cap G \neq \emptyset \text { ") } \\
= & p M_{L} \frac{\partial M_{L}}{\partial p} .
\end{aligned}
$$

Combining (7.4)-(7.7) yields

$$
M_{L} \leqq \tilde{h} \chi_{L}+M_{L}^{2}+p M_{L} \frac{\partial M_{L}}{\partial p}
$$

from which (7.2) follows by applying the "subdivision of bonds" technique to ghost bonds connecting lattice sites to the green site $g$.

Repeating the arguments of Sects. 4 and 5, using inequalities (7.1) and (7.2) in place of (3.1) and (3.2), we can prove the site percolation versions of Theorems 1.1 and 1.2.

Theorem 7.3. For a translation invariant independent site percolation model on $\mathbb{Z}^{d}$,

$$
p_{T}=p_{H} \quad\left(\equiv p_{c}\right),
$$

and

$$
M\left(p_{c}, h\right) \geqq\left(\frac{1-M\left(p_{c}, h\right)}{p_{c}|\mathcal{N}|}\right)^{1 / 2} h^{1 / 2} .
$$

Remark. The explicit bound which proves (7.8) is

$$
M(p, 0)-M\left(p_{T}, 0\right) \geqq\left[1-M\left(p_{T}, 0\right)\right]\left(\frac{p-p_{T}}{p}\right)
$$

for $p \geqq p_{T}$.

Finally, let us mention that the extrapolation principles of Sect. 6 may be derived also from (7.1) for site percolation models. In particular, the critical exponent inequality (6.1) (Corollary 6.3) still holds.

\section{Further Extensions}

The results of this paper can be applied to study other systems of interest in statistical mechanics. In this section we briefly discuss weakly nonhomogeneous percolation systems, the contact process, and a class of Ising type spin models. 


\subsection{Nonhomogeneous Percolation Models}

The differential inequalities (3.1) and (3.2) upon which the above analysis rested have interesting extensions to the nonhomogeneous case. If we repeat the arguments found in the proof of Propositions 3.1 and 3.2, we find

$$
\frac{\partial M_{x}}{\partial \beta} \leqq|J| \hat{M} \chi_{x}
$$

and

$$
M_{x} \leqq h \chi_{x}+M_{x}^{2}+\beta \hat{M} \frac{\partial M_{x}}{\partial \beta},
$$

where

$$
\begin{gathered}
M_{x}=M_{x}(\beta, h)=\operatorname{Prob}(C(x) \cap G \neq \emptyset), \\
\hat{M}=\sup _{y} M_{y}, \quad \chi_{x}=\frac{\partial M_{x}}{\partial h},
\end{gathered}
$$

and

$$
|J|=\sup _{y}\left\{\sum_{z}\left(J_{y, z}+J_{y \rightarrow z}\right)\right\} .
$$

It is not clear to us whether the general relations (8.1) and (8.2) always have useful implications. However, in the weakly nonhomogeneous case where there exists a constant $C(<\infty)$ such that $M_{0} \leqq C M_{x}$ for all $x$ accessible from the origin, (8.1) and (8.2) can be used to prove the equality of the critical points (5.17) as well as the critical exponent bounds (4.10) and (5.8). In particular, bond percolation models with periodic couplings are weakly nonhomogeneous and this observation solves the third of Kesten's Unsolved Problems [20].

Even if a system is not homogeneous it may be stochastically invariant, i.e., generated randomly by a translation invariant law. It is an easy exercise, which we leave to the reader, to show that our main conclusions also apply to stochastically invariant systems obtained by independent removal of lattice bonds. Since the more general nonhomogeneous stochastically invariant case is still not well understood, let us mention here that (3.1) has the following inhomogeneous version:

$$
M_{x} \leqq h \frac{\partial M_{x}}{\partial h}+M_{x}^{2}+\sum_{u, v} M_{v}\left(J_{u \rightarrow v} \frac{\partial}{\partial J_{u \rightarrow v}}+J_{u, v} \frac{\partial}{\partial J_{u, v}}\right) M_{0} .
$$

If the ghost field is also made inhomogeneous, the term $h \frac{\partial M_{x}}{\partial h}$ should be replaced by $\sum_{y} h_{y} \frac{\partial M_{x}}{\partial h_{y}}$, and instead of (3.1) we have

$$
\frac{\partial M_{x}}{\partial J_{u \rightarrow v}} \leqq \beta M_{v} \frac{\partial M_{x}}{\partial h_{u}},
$$


and

$$
\frac{\partial M_{x}}{\partial J_{u, v}} \leqq \beta\left[M_{v} \frac{\partial M_{x}}{\partial h_{u}}+M_{u} \frac{\partial M_{x}}{\partial h_{v}}\right] .
$$

At this time we know of no further implications of (8.3) and (8.4).

\subsection{The Contact Process}

The contact process is a dynamic interacting particle system which may be thought of as modeling the spread of an "infection." The medium through which the infection spreads (stochastically) is $\mathbb{Z}^{d}$ and the infection at each site is described by a binary random variable. That is, each site is either infected or not; there is no intermediate degree of infection.

For the discrete time contact process, the configurations are updated in unit time intervals. In each time step every infected site $x$ has some fixed probability $p \in(0,1)$ of healing itself and probability $1-e^{-\beta J_{x \rightarrow y}}$ of infecting any other site $y$. We allow both short and long range couplings $J_{x \rightarrow y}$ as in Sect. 2.1.

In the more commonly studied continuous time contact processes, the infection at $x$ heals with an exponential waiting time which, by adjusting the time scale, may be assumed to be one. The infected site $x$ also infects other sites $y$ with exponential rates $\beta J_{x \rightarrow y}$. The analysis of this paper has a straightforward application to discrete time processes, while some additional technical considerations seem to be required for continuous time processes.

One is interested in the stationary states (time invariant probability measures on the space of configurations) and in the nature of convergence to equilibrium. Certain domination arguments show that all of the stationary measures are bracketed between two (possibly equal) extremal states. One of these states, $\delta_{0}$, is concentrated on the totally healed configuration and the other, $\mu_{\beta}$, is the measure obtained by taking the infinite time limit starting from the totally infected configuration. For $\beta$ sufficiently small, every initial measure converges exponentially fast (on local events) to $\delta_{0}$ which is the unique stationary state. At the other extreme, when $\beta$ is large the process is no longer completely ergodic and $\mu_{\beta} \neq \delta_{0}$.

We define the order parameter of the contact process to be the density of the infected sites with respect to $\mu_{\beta}$ :

$$
\begin{aligned}
M(\beta) & =\mu_{\beta}(\{0 \text { is infected }\}) \\
& =\lim _{s \rightarrow-\infty} \operatorname{Prob}(0 \text { is infected at } t=0 \mid \text { every site was infected at } t=s) .
\end{aligned}
$$

It is readily observed that $M$ is an increasing function of $\beta$ and that $M \equiv 0$ if and only if the process is completely ergodic. The critical value of the infection parameter is defined to be

$$
\beta_{c}=\inf \{\beta: M(\beta)>0\} .
$$

Although each "subcritical" (i.e., $\beta<\beta_{c}$ ) contact process is completely ergodic, the convergence of arbitrary initial measures to $\delta_{0}$ need not a priori be exponentially rapid. However, as described below, our analysis shows that the 
infection dies out exponentially fast for every subcritical process (and that it dies out no faster than by a power law in the critical, i.e., $\beta=\beta_{c}$, case).

To make this statement precise, we introduce the variable

$$
T=\sum_{x} t_{x}
$$

where $t_{x}$ is the total length of time that the site $x$ is infected:

$$
t_{x}=\int_{0}^{\infty} d t I[x \text { is infected at time } t] .
$$

Letting $v_{0}$ denote the measure describing the contact process which begins at time $t=0$ with only the origin infected, we define

$$
\chi_{\text {total }}(\beta)=\langle T\rangle_{v_{0}}=\sum_{x}\left\langle t_{x}\right\rangle_{v_{0}} .
$$

Here $\langle-\rangle_{v_{0}}$ denotes the expectation value with respect to $v_{0}$.

We are now ready to state our results. In the remainder of this section we comment briefly on the arguments which we use.

Theorem 8.1. Discrete time contact processes have the following properties.

i) For any subcritical $\left(\beta<\beta_{c}\right)$ process,

$$
\chi_{\text {total }}(\beta)<\infty \text {. }
$$

Furthermore,

$$
v_{0}(\{T>\tau\}) \leqq \sqrt{\frac{e}{\tau}} e^{-\tau / 2 \chi_{\text {tota } 1}^{2}}
$$

and, in the nearest neighbor contact process,

$$
v_{0}\left(\left\{t_{x}>0\right\}\right) \leqq \text { const } e^{-\|x\| / \chi_{\text {total }}}
$$

where $\|x\|$ is the $\ell_{1}$ norm: $\|x\|=\sum_{i=1}^{d}\left|x_{i}\right|$.

ii) For any supercritical $\left(\beta>\beta_{c}\right)$ process,

$$
M(\beta)-M\left(\beta_{c}\right) \geqq\left[1-M\left(\beta_{c}\right)\right]\left(\frac{\beta-\beta_{c}}{\beta}\right) .
$$

iii) For the critical $\left(\beta=\beta_{c}\right)$ process,

$$
\limsup _{\tau \rightarrow \infty} v_{0}(\{T>\tau\}) \tau^{1 / 2} \geqq \text { const }>0 .
$$

Remarks. 1) Statement (8.8) may be viewed as the heart of the matter. For one dimensional nearest neighbor processes (continuous as well as discrete time), the results in part i) of Theorem 8.1 were proven by Griffeath [21].

2) The analysis of Griffeath and our proof of Theorem 8.1 both make use of a relation between the contact process and oriented percolation which, for a discrete time process, is seen by considering the space-time picture of the process in $\mathbb{Z}^{d} \times \mathbb{Z}$. From this viewpoint the histories of infections are the clusters of an oriented percolation model called the percolation substructure of the contact process. The order parameter $M(\beta)$ is the probability that the cluster of the origin extends back 
to the $t=s$ hyperplane as $s \rightarrow-\infty$ in the percolation model. Hence $M(\beta)$ is the percolation density. Similarly, $\chi_{\text {total }}$ is the expected cluster size. It is interesting to note that Griffeath proved the equality $p_{T}=p_{H}$ for two dimensional nearest neighbor directed percolation models (by arguments very different than those used here).

3) The proof is as follows. Our analysis of oriented percolation models shows that $\beta_{c}=\beta_{c}^{\prime}$, where

$$
\beta_{c}^{\prime}=\sup \left\{\beta: \chi_{\text {total }}(\beta)<\infty\right\} .
$$

Once it is known that $\chi_{\text {total }}$ is finite throughout the entire subcritical regime, the arguments of Aizenman and Newman [8] may be used to prove (8.9) and hence the exponential convergence of initial measures to $\delta_{0}$. The bound (8.10) is proven by means of the Hammersley inequality $[7,8]$, which is often referred to as the percolation version of the Simon-Lieb inequality. The bounds (8.11) and (8.12) correspond to our critical exponent bounds on $\widehat{\beta}$ and $\delta$.

4) We expect the natural extension of Theorem 8.1 to be valid also for continuous time contact processes, but we are unable to prove this at the present. Our difficulty lies in the need to control the effects of short time fluctuations for the continuum extension of (3.2). However, the validity of (3.1) in the continuum is easily verified.

5) It is interesting to note that one learns here about the standard contact process by imbedding it in a larger class of process. In this context, the parameter $h$ appears as a spontaneous infection rate.

\subsection{Ising Type Models}

As mentioned in the introduction, this work was stimulated in part by some related works on Ising models. It may therefore be interesting to note that the percolation results presented here have in turn inspired new results for these ferromagnetic models. Specifically, in the companion paper with R. Fernández [22] we show that the order parameter $M$ in the Ising model satisfies

$$
M \leqq h \chi+M^{3}+\beta M^{2} \frac{\partial M}{\partial \beta} .
$$

Since (8.13) is stronger than (3.2) and because the Ising type order parameters also satisfy (3.1), it follows that the analysis of Sects. 4 and 5 is immediately applicable to these models. In this fashion one obtains a proof of the equality of $\beta_{T}$ and $\beta_{H}-$ or $\beta_{c}$ and $\beta_{m}$ (for spontaneous magnetization) as they are designated in [22].

A stronger inequality than (3.2), for instance (8.13), would imply stronger bounds than (4.10) and (5.8) on the critical exponents $\delta$ and $\hat{\beta}$. In view of the wider applicability of the method of Sects. 4 and 5, we remark that the main result on the equality of the critical points would have followed had it only been known that, along with some simple monotonicity properties and (3.1), $M$ obeys the generalization of (3.2) [and (8.13)] given in (5.9):

$$
M \leqq h \chi+M f(M)+a M^{\theta} \frac{\partial M}{\partial \beta} .
$$


The critical exponent bounds which would follow from the above statement are $\delta \geqq 1+\theta$, and $\hat{\beta} \leqq \frac{1}{\theta}$.

\section{Appendix. The Infinite Volume Limit; Continuity of $M(\beta, h)$}

In Sect. 2.3 we left two claims unproven. These claims are generally known to be true for finite range unoriented models. However, since some of the simple proofs do not apply to either the long range case, or to the partially oriented percolation models, we shall supply here arguments which deal with the general case. The two issues which we now address are the convergence of the finite volume quantities (in particular, $M_{L}$ ) to their full volume analogues and the continuity of the order parameter in $\beta$. The first result (called Proposition 2.3 in Sect. 2) was an essential ingredient in our analysis. The second result (Lemma 2.6) is not used in this paper but is included for the sake of completeness. We restate both of these propositions here.

Proposition A.1. For any $\beta \geqq 0$ and $h>0$,

$$
M_{L}(\beta, h) \underset{L \rightarrow \infty}{\longrightarrow} M(\beta, h) \quad \text { and } \quad \chi_{L}(\beta, h) \underset{L \rightarrow \infty}{\longrightarrow} \chi(\beta, h) .
$$

Proposition A.2. (i) $M$ is a continuous function of $\beta$ for $h>0$ and $\beta \geqq 0$ and - in the unoriented case - also for $h=0$ and $\beta \neq \beta_{H}$.

(ii) In finite range models, $M$ is analytic in $\beta$ for $h>0$.

Both propositions make use of the following two estimates on $P_{n}(\beta)$.

Lemma A.3. For every $L$,

$$
\begin{aligned}
\operatorname{Prob}_{L}(|C(0)| & =n, \operatorname{diam} C(0) \geqq D) \\
& \leqq \beta\left(\sum_{k=1}^{n-1}(\beta|J|)^{k-1}\right)_{x:\|x\| \geqq D /(n-1)}\left(J_{0, x}+J_{0 \rightarrow x}\right),
\end{aligned}
$$

where $\operatorname{diam} A=\max \{\|x-y\|: x, y \in A\}$ and, for notational convenience, distances are measured using the $\ell_{\infty}$ norm: $\|x\|=\max \left\{\left|x_{i}\right|: i=1, \ldots, d\right\}$. The same bound is also satisfied ( for $n<\infty)$ when Prob $_{L}$ is replaced with the full volume measure Prob.

Remark. Note that by the summability of $J_{b}$, the right-hand side of (A.1) vanishes as $D \rightarrow \infty$, uniformly in $L$ and uniformly on compacta in $\beta$.

Proof. Suppose that in a given configuration the cluster of the origin contains exactly $n$ sites. By disregarding occupied bonds which contribute to loops in this cluster it is possible to reduce the bond set of the cluster to a spanning tree of $n-1$ bonds. If the diameter of the cluster is at least $D$ then some bond in the tree must have length at least $D /(n-1)$. Thus there must be a (nonrepeating) walk along the spanning bonds which starts at the origin, proceeds along bonds in the allowed direction(s) and reaches this long bond in $n-1$ steps or less. So

$$
\begin{aligned}
& \operatorname{Prob}_{L}(\mid C(0)=n, \operatorname{diam} C(0) \geqq D) \\
& \quad \leqq \sum_{k=1}^{n-1} \sum_{i=1}^{k-1}\left(\sum_{b_{i}} \operatorname{Prob}_{L}\left(n_{b_{i}}=1\right)\right)_{b_{k}:\left\|x_{k-1}-x_{k}\right\| \geqq D /(n-1)} \operatorname{Prob}_{L}\left(n_{b_{k}}=1\right),
\end{aligned}
$$


where the bond $b_{1}$ is either the unoriented bond $\left\{0, x_{1}\right\}$ or the oriented bond $\left(0, x_{1}\right)$ and the succeeding bonds $b_{i}$ are either of the form $\left\{x_{i-1}, x_{i}\right\}$ or $\left\{x_{i-1}, x_{i}\right)$. Using $1-e^{-x} \leqq x$, we replace (A.2) with

$$
\begin{aligned}
\operatorname{Prob}_{L}(|C(0)| & =n, \operatorname{diam} C(0) \geqq D) \\
& \leqq \beta \sum_{k=1}^{n-1}\left(\beta\left|J^{(L)}\right|\right)^{k-1} x_{k}: \| x_{k-1}-\sum_{x_{k} \| \geqq D /(n-1)}\left(J_{0, x}^{(L)}+J_{0 \rightarrow x}^{(L)}\right) .
\end{aligned}
$$

In the first sum in (A.3) observe that $\left|J^{(L)}\right|=|J|$ and in the second sum

$$
\sum_{\|x\| \geqq D /(n-1)} J_{0, x}^{(L)}=\sum_{L \geqq\|x\| \geqq D /(n-1)} \sum_{y} J_{0, x+2 L y} \leqq \sum_{\|x\| \geqq D /(n-1)} J_{0, x} .
$$

A similar bound holds for the oriented couplings.

Combining (A.4) and its oriented analogue with (A.3) proves the bound (A.1). The full volume version of (A.1) is proved by repeating the argument which leads to (A.3).

Lemma A.4. For every $D$,

$$
\operatorname{Prob}_{L}(|C(0)|=n, \operatorname{diam} C(0)<D) \underset{L \rightarrow \infty}{\longrightarrow} \operatorname{Prob}(|C(0)|=n, \operatorname{diam} C(0)<D) .
$$

Moreover, the convergence is uniform on compacta in $\beta$.

Proof. We begin by decomposing the event " $|C(0)|=n$, diam $C(0)<D$ " as follows: $\operatorname{Prob}_{L}(|C(0)|=n, \operatorname{diam} C(0)<D)$

$=\sum_{\substack{A, B: \backslash A \mid=n \\ \operatorname{diam} A<D}} \operatorname{Prob}_{L}(A=C(0), B$ is the set of occupied bonds connecting $C(0))$
$=\sum_{\substack{A, B: T A \mid=n \\ \operatorname{diam} A<D}} E_{L}(A) F_{L}(B) I[A$ is the cluster corresponding to the bond set $B]$,

where

$$
E_{L}(A)=\prod_{\substack{x \in A \\ y \in A_{L} \backslash A}} e^{-\beta\left(J_{x, y}^{(L)}+J_{x \rightarrow y}^{(L)}\right)}
$$

and

$$
F_{L}(B)=\prod_{b \in B}\left(1-e^{-\beta J_{b}^{(L)}}\right) .
$$

The same decomposition can be used for the full volume probability of the above event where the only changes in (A.6)-(A.8) are the removal of the " $L$ " subscripts and superscripts and, in (A.7), the relaxation of " $y \in \Lambda_{L} \backslash A$ " to " $y \in \mathbb{L} \backslash A$."

Upon considering the ratio of the infinite products $E_{L}$ and $E$, one finds

$$
\frac{E_{L}}{E}=\exp \left\{-\beta \sum_{\substack{x \in A \\ y \in A_{L} \backslash A \\ z \neq 0}}\left(J_{x, y+2 L z}+J_{x \rightarrow y+2 L z}\right)\right\}
$$

which implies

$$
1 \geqq \frac{E_{L}}{E} \geqq \exp \left\{-\beta|A| \sum_{x:\|x\| \geqq L}\left(J_{0, x}+J_{0 \rightarrow x}\right)\right\}
$$


Note that the right-hand side of (A.9) tends to one (uniformly on compacta in $\beta$ ) as $L \rightarrow \infty$.

Because there are only a finite number of factors in the products $F_{L}$ and $F$, and since $J_{b}^{(L)} \rightarrow J_{b}$ as $L \rightarrow \infty$ for each $b \in B, F_{L} \rightarrow F$.

Thus $E_{L}(A) \cdot F_{L}(B) \rightarrow E(A) \cdot F(B)$ as $L \rightarrow \infty$. This proves the lemma, since there are only a fixed number of pairs $(A, B)$ which make a positive contribution to the sum in (A.9).

The following result is not only the heart of the proofs of Propositions A.1 and A.2(i), but it also may be of independent interest.

Lemma A.5. Let $n$ be finite. Then

(i) $\lim _{n \rightarrow \infty} P_{n}^{(L)}(\beta)=P_{n}(\beta)$ and the convergence is uniform on compacta in $\beta$, and

(ii) $P_{n}(\beta)$ is continuous in $\beta$.

Proof. For both statements we use the decomposition

$$
P_{n}^{(L)}(\beta)=\operatorname{Prob}_{L}(|C(0)|=n, \operatorname{diam} C(0)<D)+\operatorname{Prob}_{L}(|C(0)|=n, \operatorname{diam} C(0) \geqq D) .
$$

By Lemma A.3 the second term can be made arbitrarily small; by choosing $D$ large enough, and using Lemma A.4, we see that the first term in (A.10) has the desired convergence and continuity properties.

Proof of Proposition A.1. In light of Lemma A.5(i), this is just a simple application of the Dominated Convergence Theorem.

Proof of Proposition A.2. (i) By Lemma A.5(ii), $M(\beta, h)$ is continuous in $\beta$ whenever $h$ is positive. For the unoriented $h=0$ case, see [18].

(ii) This result is well-known in percolation theory (see [23]). The proof is based on the observation that for each finite $n$ we may write $P_{n}$ as a polynomial with nonnegative coefficients in the variables $p_{b}=\operatorname{Prob}\left(n_{b}=1\right)$ and $q_{b}=1-p_{b}$. Once $P_{n}$ is written in this form, bounds on all of its derivatives are easily obtained and analyticity of $M(\beta, h)$ is not hard to show.

Acknowledgements. We wish to thank C. Newman, J. Fröhlich, A. Sokal, and J. and L. Chayes for useful discussions on their results on topics related to this work. We would like to thank M. Beals for his comments on earlier drafts of this paper and J. Lebowitz, M. Bramson, and R. Schonmann for stimulating our interest in the contact process.

\section{References}

1. Kesten, H.: The critical probability of bond percolation on the square lattice equals $1 / 2$. Commun. Math. Phys. 74, 41-59 (1980)

2. Russo, L.: On the critical percolation probabilities. Z. Wahrscheinlichkeitstheor. Verw. Geb. 56, 229-237 (1981)

3. Aizenman, M., Newman, C.M.: Discontinuity of the percolation density in one-dimensional $1 /|x-y|^{2}$ percolation models. Commun. Math. Phys. 107, 611-647 (1986)

4. Aizenman, M., Chayes, J.T., Chayes, L., Imbrie, J., Newman, C.M.: An intermediate phase with slow decay of correlations in one-dimensional $1 /|x-y|^{2}$ Ising and Potts models (in preparation) 
5. Aizenman, M.: Contribution in: Statistical physics and dynamical systems (Proceedings Kösheg 1984). Fritz, J., Jaffe, A., Szasz, D. (eds.). Boston: Birkhäuser 1985

6. Chayes, J.T., Chayes, L.: Critical points and intermediate phases on wedges of $\mathbb{Z}^{d}$. J. Phys. A (to appear)

7. Hammersley, J.M.: Percolation processes. Lower bounds for the critical probability. Ann. Math. Statist. 28, 790-795 (1957)

8. Aizenman, M., Newman, C.M.: Tree graph inequalities and critical behavior in percolation models. J. Stat. Phys. 36, 107-143 (1984)

9. Newman, C.M., Schulman, L.S.: One-dimensional $1 /|j-i|^{s}$ percolation models: The existence of a transition for $s \leqq 2$. Commun. Math. Phys. 104, 547-571 (1986)

10. Griffiths, R.B., Hurst, C.A., Sherman, S.: Concavity of magnetization of an Ising ferromagnet in a positive external field. J. Math. Phys. 11, 790-795 (1970)

11. Newman, C.M.: Shock waves and mean field bounds. Concavity and analyticity of the magnetization at low temperature. Appendix to contribution in Proceedings of the SIAM workshop on multiphase flow, G. Papanicolau (ed.) (to appear)

12. Aizenman, M., Fernández, R.: On the critical behavior of the magnetization in highdimensional Ising models. J. Stat. Phys. 44, 393-454 (1986)

13. Harris, A.B., Lubensky, T.C., Holcomb, W.K., Dasgupta, C.: Renormalization group approach to percolation problems. Phys. Rev. Lett. 35, 327-330 (1975)

14. Chayes, J.T., Chayes, L.: An inequality for the infinite cluster density in Bernoulli percolation. Phys. Rev. Lett. 56, 1619-1622 (1986)

15. Fröhlich, J., Sokal, A.D.: The random walk representation of classical spin systems and correlation inequalities. III. Nonzero magnetic field (in preparation)

16. Fernández, R., Fröhlich, J., Sokal, A.D.: Random-walk models and random-walk representations of classical lattice spin systems (in preparation)

17. Griffiths, R.B.: Correlations in Ising ferromagnets. II. External magnetic fields. J. Math. Phys. 8, 484-489 (1967)

18. Aizenman, M., Kesten, H., Newman, C.M.: Uniqueness of the infinite cluster and continuity of connectivity functions for short and long range percolation. Submitted to Commun. Math. Phys.

19. van den Berg, J., Kesten, H.: Inequalities with applications to percolation and reliability. J. Appl. Probab. 22, 556-569 (1985)

20. Kesten, H.: Percolation theory for mathematicians. Boston: Birkhäuser 1982

21. Griffeath, D.: The basic contact process. Stochastic Processes Appl. 11, 151-185 (1981)

22. Aizenman, M., Barsky, D.J., Fernández, R.: The phase transition in a general class of Isingtype models in sharp. Submitted to J. Stat. Phys.

23. Chayes, J.T., Chayes, L., Newman, C.M.: Bernoulli percolation above threshold: an invasion percolation analysis. Ann. Probab. (to appear)

Communicated by J. Fröhlich

Received September 28, 1986; in revised form October 24, 1986

Note added in proof. After submitting the manuscript we learned that the equality of $p_{T}$ and $p_{H}$ was also proven, for finite range models, by Menshikov, M.V., Molchanov, S.A., Sidovenko, S.F. (in press), by means of an independent and somewhat different argument. 